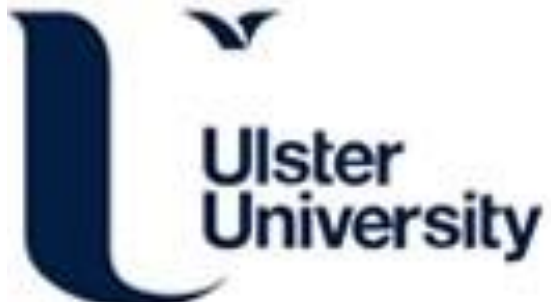

\section{A Micro-Extended Belief Rule-Based System for Big Data Multi-Class Classification Problems}

Yang, L., Liu, J., Wang, Y., \& Martinez, L. (2021). A Micro-Extended Belief Rule-Based System for Big Data Multi-Class Classification Problems. IEEE Transactions on Systems, Man, and Cybernetics: Systems, 51(1), 420-440. https://doi.org/10.1109/TSMC.2018.2872843

Link to publication record in Ulster University Research Portal

Published in:

IEEE Transactions on Systems, Man, and Cybernetics: Systems

Publication Status:

Published (in print/issue): 01/01/2021

DOI:

10.1109/TSMC.2018.2872843

Document Version

Author Accepted version

\section{General rights}

Copyright for the publications made accessible via Ulster University's Research Portal is retained by the author(s) and / or other copyright owners and it is a condition of accessing these publications that users recognise and abide by the legal requirements associated with these rights.

\section{Take down policy}

The Research Portal is Ulster University's institutional repository that provides access to Ulster's research outputs. Every effort has been made to ensure that content in the Research Portal does not infringe any person's rights, or applicable UK laws. If you discover content in the Research Portal that you believe breaches copyright or violates any law, please contact pure-support@ulster.ac.uk. 


\title{
A Micro-Extended Belief Rule-Based System for Big Data Multi-Class Classification Problems
}

\author{
Long-Hao Yang, Jun Liu, Member, IEEE, Ying-Ming Wang, and Luis Martínez, Member, IEEE
}

\begin{abstract}
Big data classification problems have drawn great attention from diverse fields and many classifiers have been developed. Among those classifiers, the extended belief rule-based system (EBRBS) has shown its potential in both big data and multi-class situations while time complexity and computing efficiency are two challenging issues to be handled in EBRBS. As such, three improvements of EBRBS are proposed firstly in the present paper to decrease the time complexity and computing efficiency of EBRBS for multi-class classification under the assumption of large amount of data, including the strategy to skip rule weight calculation, a simplified evidential reasoning algorithm, and the domain division-based rule reduction method. This turns out to be a micro version of the EBRBS, classed Micro-EBRBS. Moreover, one of commonly used cluster computing, named Apache Spark, is then applied to implement the parallel rule generation and inference schemes of the Micro-EBRBS for big data multiclass classification problems. The comparative analyses of experimental studies demonstrate that the Micro-EBRBS not only can obtain a desired accuracy, but also has the comparatively better time complexity and computing efficiency than some popular classifiers, especially for multi-class classification problems.
\end{abstract}

Index Terms-Apache Spark, Big data, Extended belief rulebased system (EBRBS), Multi-class,

Manuscript received February 2, 2018; accepted September 11, 2018. This work was supported by the National Natural Science Foundation of China (Nos. $61773123,71371053,71501047,71701050$, and 71801050), the Humanities and Social Science Foundation of the Ministry of Education under Grant (No. 14YJC630056), and the Natural Science Foundation of Fujian Province, China (No. 2015J01248). This paper was recommended by Associate Editor XXXX (Corresponding authors: Ying-Ming Wang).

L.-H. Yang is with Decision Sciences Institute, Fuzhou University, Fuzhou, PR China, and also with Department of Computer Science, University of Jaén, Jaén, Spain (e-mail: more026@ hotmail.com).

J. Liu is with School of Computing, Ulster University at Jordanstown Campus, Northern Ireland, UK (e-mail: j.liu @ulster.ac.uk).

Y.-M. Wang is with Decision Sciences Institute, Fuzhou University, Fuzhou, PR China, and also with Key Laboratory of Spatial Data Mining \& Information Sharing of Ministry of Education, Fuzhou University, Fuzhou, RP China (e-mail: msymwang@hotmail.com).

L. Martínez is with Department of Computer Science, University of Jaén, Jaén, Spain (email: martin@ujaen.es)

Color versions of one or more of the figures in this paper are available online at http://ieeexplore.iee.org.

Digital Object Identifier XXXXXXX

\section{INTRODUCTION}

CLASSIFICATION problems are the common and fundamental ones involved in various real-world applications, such as intrusion detection [1], pattern recognition [19], image processing [2], and DNA sequence classification [5]. Classification becomes much more complex under big data and multi-class situations. The former always implies a high requirement of the computing efficiency of classifiers, while the need of a cluster computing for implementing the classifiers is getting popular. The latter implies many overlaps among the data of different classes, which requires the classifiers to have a powerful ability to differentiate the class boundaries.

Among many methodologies for multi-class classification problems, such as support vector machine (SVM) [34], ensemble learning [21], and others, rule-based systems (RBSs) are one kind of useful tools and have been a popular framework for designing classifiers in past decades. Basically, these RBSs can be divided into two categories depending on the construction methods of rule bases: RBSs based on the optimization model and iterative algorithm to determine the optimal values of parameters involved [33], [36], [39], or RBSs where rules are generated from sample data without the optimal model [8], [22], [28]. While handling big data multi-class classification problems, it is obvious that the RBSs without the optimal model are a better choice because of its high computing efficiency [26].

Some popular RBSs without the optimal model include fuzzy rule-based classification system (FRBCS) based on Chi et al. algorithm (Chi-FRBCS) [8] and the extended belief rule-based system (EBRBS) proposed by Liu et al. [22], both of them are originated from the work by Wang and Mendel [31]. It is worth noting that Chi-FRBCS has been applied to deal with big data classification problems recently (see details in Section II-B). Although there are some existing works about EBRBS [6], [11], [38], [39], it has not been developed yet as an efficient classifier to deal with big data classification problems. Hence, the goal of this study is to propose a novel big data EBRBS classifier and show its performance, in terms of accuracy and efficiency, in big data multi-class classification problems.

EBRBS has shown its potential to address multi-class classification problems because of its rules with belief structure and the evidential reasoning (ER) algorithm used in the inference scheme to collectively handle multi-class information for classification [20], [23], [24], [25], however, two challenges must be addressed to handle the big data situation: 


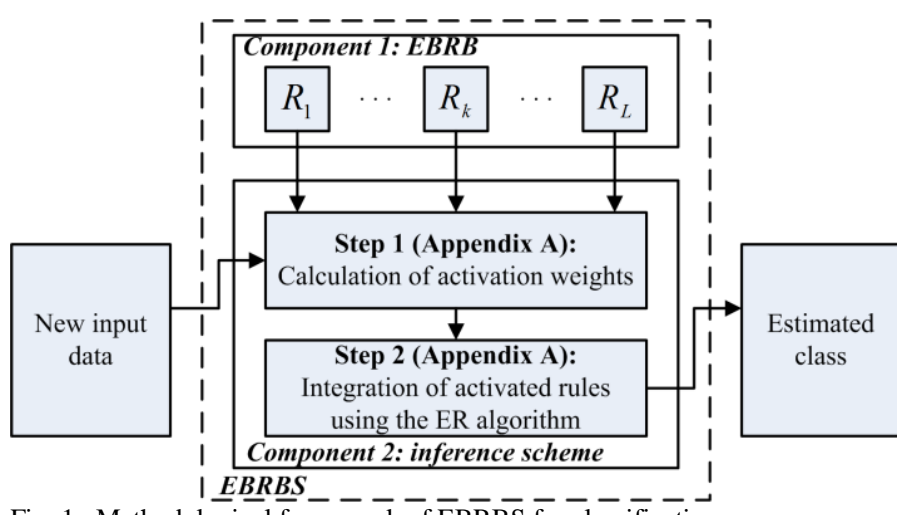

Fig. 1. Methodological framework of $\overline{\mathrm{E} B R} \overline{\mathrm{BS}} \overline{\text { for classification }}$

(1) The time complexity of the EBRBS must be reduced to ensure the high computing efficiency under big data problems.

The proposed solution in the present paper is to optimize the procedures of the EBRBS so that its time complexity can be reduced, which are mainly focused on three key procedures of the EBRBS: rule weight calculation, the ER algorithm, and the size of rule base. The corresponding improvements are proposed respectively, which forms a micro version of the EBRBS with much higher computing efficiency enables to deal with big data multi-class classification problems, called the Micro-EBRBS.

(2) The cluster computing must be applied to implement the parallel computing to improve the computing efficiency.

Chi-FRBCS classifiers usually involve the cluster computing to deal with big data classification problems [10], [26], [29]. Likewise, it is necessary to propose a solution of implementing the EBRBS classifier by using the cluster computing to handle the big data problem. Apache Spark [4] is an open-source framework that supports the processing of large datasets in a distributed computing environment and provides primitives for in-memory cluster computing and APIs in Scala, Java, and Python. As such, Apache Spark-based implementation of the parallel rule generation and inference scheme is proposed to improve the computing efficiency of Micro-EBRBS in big data situation.

To verify the effectiveness and computing efficiency of the Micro-EBRBS, three experiments based on 14 classification datasets, in which 4 of these datasets have relatively large number of data, are carried out to test the performance of the Micro-EBRBS. Two main aspects, namely accuracy and computing time, are used to compare the Micro-EBRBS with the EBRBS, the conventional FRBCS and machine-learning classifiers, and the big data FRBCS classifiers.

The remainder of this paper is organized as follows: Section II briefly reviews the background and challenges of the EBRBS for classification. Section III introduces the Micro-EBRBS for big data multi-classification problems. Section IV discusses experiments to demonstrate the performance of the MicroEBRBS, and the paper is concluded in Section V.

\section{BACKGROUND AND CHALLENGES}

In this section, the EBRBS for classification problems is reviewed firstly to provide the basic knowledge of this study.

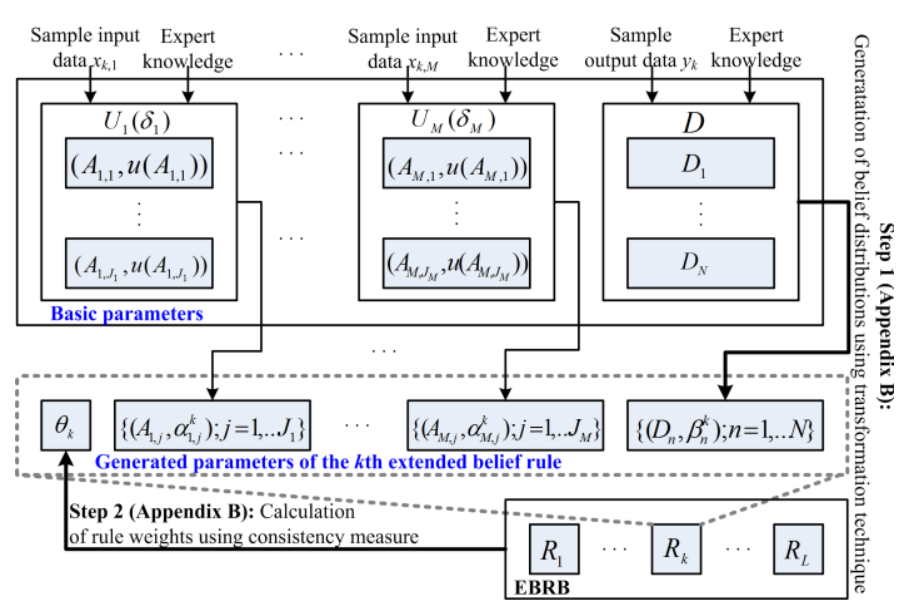

Fig. 2. Rule generation scheme of EBRBS

Secondly, the related works of the Chi-FRBCS for big data problems are reviewed for the sake of comparison with the proposed Micro-EBRBS. Finally, the time complexity of the EBRBS is discussed to clarify the challenges to face for the novel classifier under big data and multi-class situation.

\section{A. EBRBS for classification problems}

An EBRBS consists of two components: the extended belief rule base (EBRB) and the inference scheme. The former can be regarded as a knowledge base to store a set of rules with uncertainty. The latter provides an inference engine to infer new results based on the interaction of test input and the EBRB.

Basically, an EBRB for classification problems includes $M$ antecedent attributes and one consequent attribute, in which each antecedent attribute $U_{i}(i=1, \ldots, M)$ has an attribute weight $\delta_{i}\left(0<\delta_{i} \leq 1\right)$ and $J_{i}$ reference values $A_{i, j}\left(j=1, \ldots, J_{i}\right)$ which are used as the discrete and representative evaluation grades for describing the $i$ th antecedent attribute; and the consequent attribute $D$ has $N$ classes $D_{n}(n=1, \ldots, N)$. Thus, the $k$ th $(k=1, \ldots$, $L$ ) extended belief rule (EBR) $R_{k}$ in the EBRB can be written as:

$$
\begin{aligned}
R_{k} & \text { IF } U_{1} \text { is }\left\{\left(A_{1, j}, \alpha_{1, j}^{k}\right) ; j=1, \ldots, J_{1}\right\} \wedge \cdots \wedge U_{M} \text { is } \\
& \left\{\left(A_{M, j}, \alpha_{M, j}^{k}\right) ; j=1, \ldots, J_{M}\right\}, \text { THEN } D \text { is } \\
& \left\{\left(D_{n}, \beta_{n, k}\right) ; n=1, \ldots, N\right\}, \text { with } \theta_{k} \text { and }\left\{\delta_{1}, \ldots, \delta_{M}\right\}
\end{aligned}
$$

where $\alpha_{i, j}^{k}\left(0 \leq \alpha_{i, j}^{k} \leq 1\right)$ and $\beta_{n, k}\left(0 \leq \beta_{n, k} \leq 1\right)$ are the belief degree to the reference value $A_{i, j}$ and the class $D_{n}$ in the $k$ th rule with $\sum_{j=1}^{J_{i}} \alpha_{i, j}^{k} \leq 1$ and $\sum_{n=1}^{N} \beta_{n, k} \leq 1 ;\left\{\left(A_{i, j}, \alpha_{i, j}^{k}\right) ; j=1, \ldots, J_{i}\right\}$ and $\left\{\left(D_{n}, \beta_{n, k}\right) ; n=1, \ldots, N\right\}$ are the belief structure embedding in the $k$ th rule; $\theta_{k}\left(0<\theta_{k} \leq 1\right)$ is the weight of the $k$ th rule.

Remark 1: By assuming that: (1) $A_{i, j}\left(i=1, . ., M ; j=1, \ldots, J_{i}\right)$ is a linguistic label modeled by a triangular membership function; (2) $\alpha_{i, j}^{k}=1(k=1, \ldots, L)$ and $\alpha_{i, t}^{k}=0\left(t=1, \ldots, J_{i} ; t \neq j\right)$; (3) $\beta_{n, k}=1$ and $\beta_{n, s}=0(s=1, \ldots, N ; s \neq n)$; (4) $\delta_{i}=1(i=1, \ldots, M)$, the $k$ th EBR becomes a fuzzy rule in the Chi-FRBCS [29]:

$$
\begin{gathered}
R_{k}: \text { IF } U_{1} \text { is } A_{1, j} \wedge \cdots \wedge U_{M} \text { is } A_{M, j}, \\
\quad \text { THEN D is } D_{n} \text {, with } \theta_{k}
\end{gathered}
$$




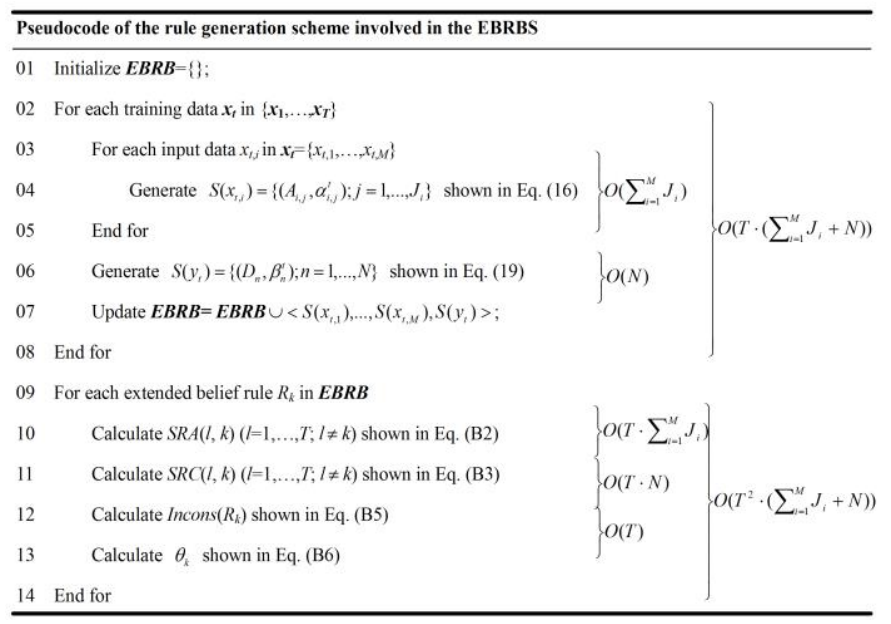

Fig. 3. Pseudocode and time complexity of the rule generation scheme

It is clear from the comparison of Eqs. (1) and (2) that the fuzzy rule is a special case of the EBR. Moreover, the EBR is more flexible to express multi-class information under uncertainty and incompleteness, e.g., $\left\{\left(D_{1}, 0.6\right),\left(D_{2}, 0.4\right)\right\}$ means $60 \%$ sure that the class is $D_{1}, 40 \%$ sure that it is $D_{2}$.

$\sum_{j=1}^{J_{i}} \alpha_{i, j}^{k}=0.9$ means $100 \%-90 \%=10 \%$ ignorance in the $i$ th antecedent attribute of the $k$ th EBR.

Based on the above EBRB, the ER algorithm based inference scheme is applied to integrate EBRs to produce estimated classes, i.e., the integrated result is belief distribution $\left\{\left(D_{1}\right.\right.$, $\left.0.4008),\left(D_{2}, 0.4275\right),\left(D_{3}, 0.1718\right)\right\}$ and finally produces the class $D_{2}$ as the output. A simple methodological framework of the EBRBS for classification is shown in Fig. 1 and the detailed step procedure can be referred to [22]. Additionally, the rule generation scheme is an indispensable part of the EBRBS and illustrated in Fig. 2.

As shown in Fig. 2, there are two kinds of parameters involved in the rule generation scheme of the EBRB. The first one is named as the basic parameters, including attribute weights, reference values and utility values of antecedent attributes, and classes of the consequent attribute. All these basic parameters are always determined by using expert knowledge. The second one is the generated parameters, including rule weights and belief distributions of antecedent and consequent attributes. All these generated parameters have to be initialized according to the sample input-output data and the basic parameters, including two steps: 1) generation of belief distributions for antecedent and consequent attributes using transformation techniques; and 2) calculation of rule weights using consistency measures. The detailed description of those steps can be found in Appendix B.

\section{B. Chi-FRBCS in big data classification problems}

FRBCSs are popular methods for classification problems with many versions developed so far, e.g., Chi-FRBCS [8], structural learning algorithm on vague environment (SLAVE) [15], fuzzy hybrid genetic-based machine learning algorithm (FH-GBML) [18], fuzzy unordered rule induction algorithm (FURIA) [16], and fuzzy association rule-based classification

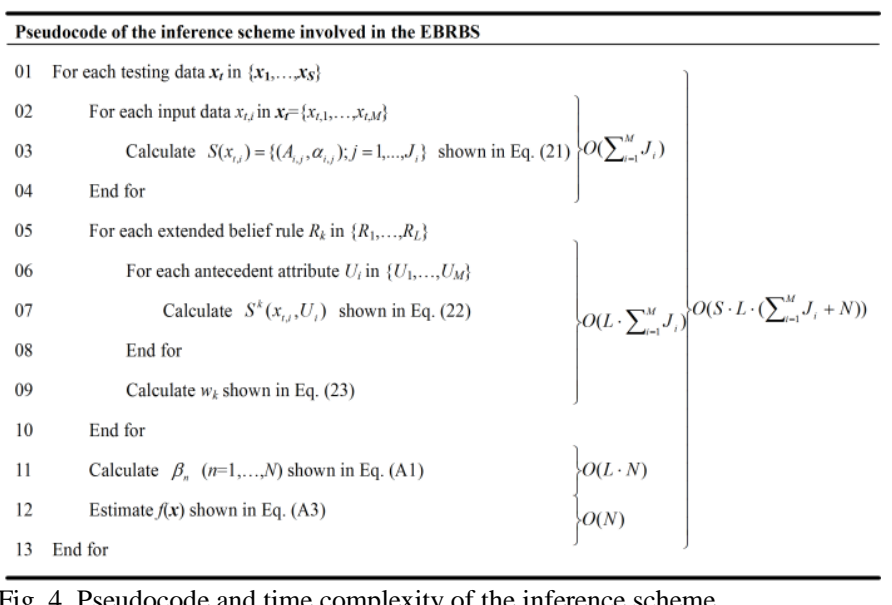

Fig. 4. Pseudocode and time complexity of the inference scheme

method for high-dimensional problems (FARC-HD) [3]. However, considering the limitations of standard fuzzy rule base learning approaches for large number of samples, the ChiFRBCS was recognized by many researchers as the suitable FRBCS to handle big data classification problems [26], [29].

In the last few years, several big data classifiers based on the Chi-FRBCS have been proposed and made use of the Apache Hadoop to deploy the distributed system. For example, Lopez et al. [26] proposed the first FRBCS capable of addressing big data and imbalance datasets, called Chi-FRBCS-BigDataCS, which utilized the Apache Hadoop to distribute the computational operations of Chi-FRBCS and also included cost-sensitive learning techniques to address imbalanced big data. After that, Rio et al. [29] developed a more general big data classifier based on the Chi-FRBCS and Apache Hadoop, called Chi-FRBCS-BigData, which includes two versions: Chi-FRBCS-BigData-Max and Chi-FRBCS-BigData-Ave. Both show the ability to deal with big data problems providing competitive results and reasonable computing efficiencies. Fernandez et al. [12] studied the relationship between the granularity and data scattering for Chi-FRBCS in big data classification problems and made use of Chi-FRBCS-BigData to accomplish their analysis. Later on, Fernandez et al. [13] carried out many experimental studies regarding the use of ChiFRBCS-BigData to analyze the differences in performance with respect to the lack of data for the learning stage, how rules are distributed among Maps, and their influences on the classification stage. Elkano et al. [10] proposed a global version of Chi-FRBCS-BigDataCS in order to address the problem of previous big data classifiers that it would become less accurate when more computing nodes are added into the cluster.

The above literatures review shows many potential applications of Chi-FRBCS in big data classification problems. However, they were mainly focused on two-class classification problems. Although the decomposition strategies [27], such as One-Versus-One (OVO) and One-Versus-All (OVA) schemes, can be used to decompose a multi-class problem into multiple two-class problems, it is unavoidable to cause the increase of the time complexity. Considering that the EBRBS has an effective rule representation scheme better than the one in ChiFRBCS owning to embedding the belief structure into both 


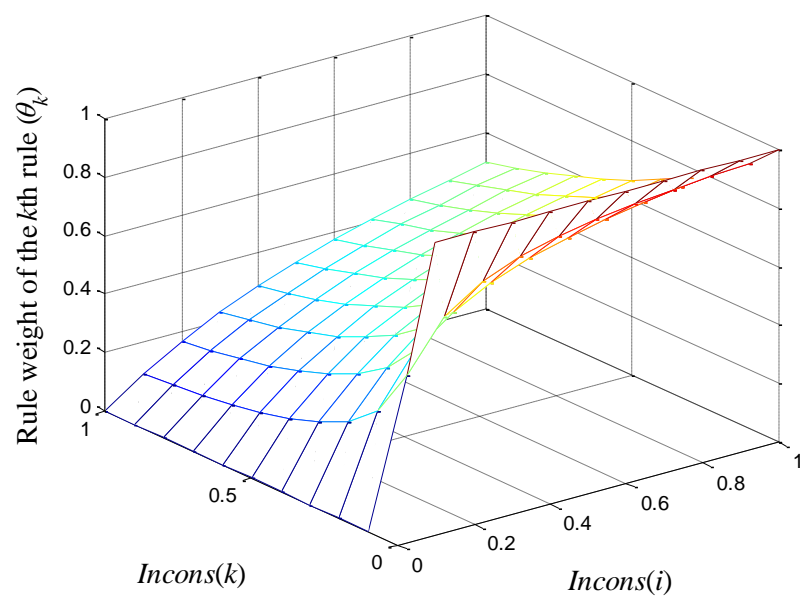

Fig. 5. Relationship between the rule weights and their inconsistency degrees

antecedent and consequent attributes, the present work aims to propose a novel classifier based on the EBRBS for big data problems in comparison with Chi-FRBCS in terms of accuracy and efficiency.

\section{Challenges of EBRBS for big data problems}

Due to the importance of computing efficiency in big data problems, in the context of the EBRBS, the time complexity of the rule generation and inference scheme are analyzed in this subsection. For the discussion purposes, Figs. 3 and 4 provide the pseudocode and the time complexity of those two schemes involved in the EBRBS, respectively.

From Fig. 3, the time complexity of generating belief distributions shown in the 1 st line to the 8 th line is $O\left(T \times\left(\sum_{i=1, \ldots}\right.\right.$, $\left.{ }_{M} J_{i}+N\right)$ and the time complexity of calculating rule weights shown in the 9th line to the 14th line is $O\left(T^{2} \times\left(\sum_{i=1, \ldots, M} J_{i}+N\right)\right.$, where $T$ is the number of sample data, $M$ is the number of antecedent attributes, $J_{i}$ is the number of reference values in the $i$ th antecedent attribute, and $N$ is the number of classes. Clearly, for the rule generation scheme of the EBRBS, the calculation of rule weights requires the most computing time and sometimes it would be unacceptable while there are large amounts of sample data. For example, the dataset Poker has 1,025,010 samples (i.e., $T=1,025,010), 10$ attributes (i.e., $M=10$ ), and 10 classes (i.e., $N=10$ ). If the number of reference values is assumed to be 3 for each attribute (i.e., $J_{i}=3 ; i=1, \ldots, M$ ) and the computing time of each operation is $10^{-6}$ second, then the total computing time of generating rule weights is $11,673.8$ hours.

From Fig. 4, the time complexity of the inference scheme is $O\left(S \times L \times\left(\sum_{i=1, \ldots, M} J_{i}+N\right)\right.$, where $S$ is the number of test data, $L$ is the number of EBRs in the EBRB. Considering that one EBR is directly transformed from one sample data [22], so $T=L$. Therefore the time complexity of the inference scheme can be expressed as $O\left(S \times T \times\left(\sum_{i=1, \ldots, M} J_{i}+N\right)\right.$. Obviously, the computing time would also be unacceptable while there is large amount of sample data involved in the rule generation scheme, i.e., while the 10-fold cross validation is utilized to test the dataset Poker, the number of sample data and test data is therefore 922,509 and 102,501, respectively. Finally, the computing time of the

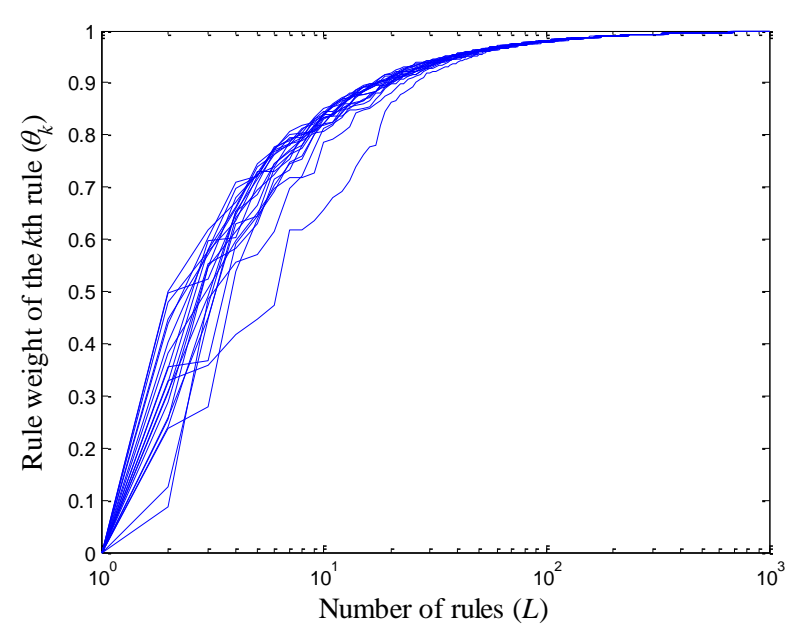

Fig. 6. Relationship between the rule weights of $R_{k}$ and the number of rules

inference scheme is $1,050.6$ hours.

The above discussions clearly show that although the EBRBS is the RBS without the optimal model, the timeconsuming process found in the calculation of rule weights and the inference scheme would be serious challenges while the EBRBS is applied to address the classification problem with a large amount of data. Therefore, the present work aims to address these challenges.

\section{A NOVEL EBRBS FOR BIG DATA MULTI-CLASS CLASSIFICATION PROBLEMS}

According to the challenges pointed out in Section II-C, the possible approaches are investigated to reduce the time complexity of the rule generation and the inference schemes, followed by a new rule reduction method to downsize the EBRB. Based on these achievements, a novel EBRBS and its Apache Spark-based implementation are developed to deal with big data multi-class classification problems.

\section{A. Analysis of rule weight calculation and the ER algorithm involved in EBRBS}

In order to reduce the time complexity of the EBRBS, the properties of the rule weight calculation and the ER algorithm are investigated as follows.

Theorem 1. The rule weight of each EBR will approximate to 1 while using large number of sample data to generate EBRs.

Proof. Suppose there are $L$ EBRs. Based on the calculation of rule weights in Eq. (B6) in Appendix B, we can get the first order partial derivative of the rule weight $\theta_{k}(k=1, \ldots, L)$ with respect to the inconsistency degree $\operatorname{Incons}\left(R_{k}\right)$ as follows:

$$
\begin{aligned}
\frac{\partial \theta_{k}}{\partial \operatorname{Incons}\left(R_{k}\right)} & =-\frac{\sum_{l=1}^{L} \operatorname{Incons}\left(R_{l}\right)-\operatorname{Incons}\left(R_{k}\right)}{\left[\operatorname{Incons}\left(R_{k}\right)+\sum_{l=1, l \neq k}^{L} \operatorname{Incons}\left(R_{l}\right)\right]^{2}} \\
& =-\frac{\sum_{l=1, l \neq k}^{L} \operatorname{Incons}\left(R_{l}\right)}{\left[\operatorname{Incons}\left(R_{k}\right)+\sum_{l=1, l \neq k}^{L} \operatorname{Incons}\left(R_{l}\right)\right]^{2}}<0
\end{aligned}
$$

It follows that $\theta_{k}$ decreases while its $\operatorname{Incons}\left(R_{k}\right)$ increases. In 

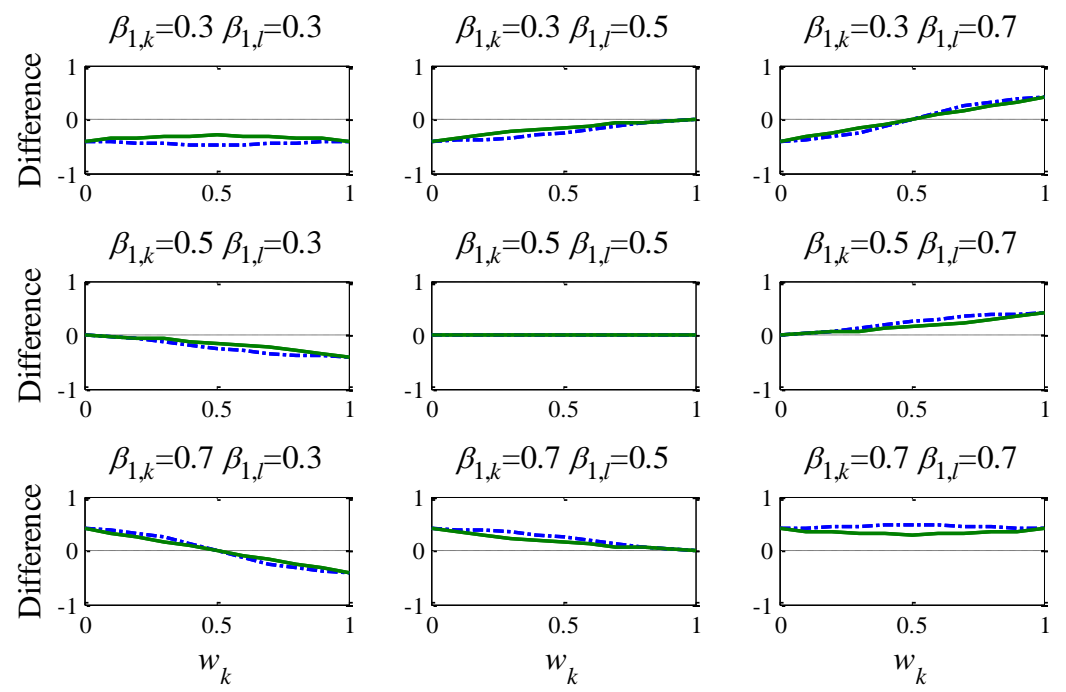

Fig. 7. Comparison of the ER-C and ER algorithms (The full line denotes $d^{E R-C}$ and the dotted line denotes $d^{E R}$ )

other words, when $\operatorname{Incons}\left(R_{k}\right)$ is equal to $1, R_{k}$ has a minimum rule weight. This makes sense that if this rule causes the contradiction, then it will be useless.

Apparently, when the number of rules is increasing, the inconsistency degree of each rule is also increasing. It follows that when $L$ is approaching to $\infty$, the inconsistency degree of each rule is approaching to 1 . Without loss of generality, we consider the rule weight of $R_{k}$ as follows:

$$
\theta_{k}=\lim _{L \rightarrow+\infty}\left[1-\frac{\operatorname{Incons}\left(R_{k}\right)}{\sum_{l=1}^{L} \operatorname{Incons}\left(R_{l}\right)}\right]=1-\lim _{L \rightarrow+\infty} \frac{1}{L}=1
$$

Now that $L$ is equal to the number of sample data, this concludes the proof.

Example 1. Suppose there are $L$ EBRs and two different EBRs, namely the $k$ th rule and the $i$ th rule $(k, i \in\{1, \ldots, L\} ; i \neq k)$ with their inconsistency degree $\operatorname{Incons}\left(R_{k}\right)$ and $\operatorname{Incons}\left(R_{i}\right)$, respectively. The relationship between rule weights and inconsistency degrees can be shown in Fig. 5: while Incons $(i)$ is fixed, $\theta_{k}$ increases with the decrease of $\operatorname{Incons}(k)$. Moreover, while $\operatorname{Incons}(k)=1$, the $k$ th rule has a minimum rule weight.

Secondly, assume that $\operatorname{Incons}\left(R_{k}\right)=1(k \in\{1, \ldots, L\})$ and the inconsistency degree of other rules are set by using random values. Then the relationship between $\theta_{k}$ and $L$ (from 1 to 1000) is shown in Fig. 6, in which each line (twenty lines in total) denotes an independent experiment of using random values to determine $\theta_{i}(i=1, \ldots, L ; i \neq k)$. From Fig. $6, \operatorname{Incons}(k)=1$ leads to the minimum rule weight for the $k$ th rule. However, with the increase of $L$, it turned out that $\theta_{k}$ approximates to 1 .

Remark 2: From Theorem 1, it is unnecessary to calculate each rule weight because its value will approximate to 1 while a large number of data are used to generate EBRs. As a result, the time complexity of the rule generation scheme is reduced from $O\left(T^{2} \times\left(\sum_{i=1, \ldots, M} J_{i}+N\right)\right.$ to $O\left(T \times\left(\sum_{i=1, \ldots, M} J_{i}+N\right)\right.$.

Theorem 2. For classification problems, the following ER algorithm for classification (denoted as ER-C) is the same as the analytical ER algorithm shown in Eq. (A1) in Appendix A.

$$
\beta_{n}^{E R-C}=\prod_{k=1}^{L}\left(w_{k} \beta_{n, k}+1-w_{k} \sum_{i=1}^{N} \beta_{i, k}\right)
$$

Proof. Suppose a classification problem has $N$ classes and the $n$th $(n=1, \ldots, N)$ class is denoted as $D_{n}$. Assume the estimated class of the EBRBS is the $n$th class for the test input data $\boldsymbol{x}$. Hence, according to Eq. (A3) in Appendix A, we have

$$
\beta_{n}>\beta_{t} ; t=1, \ldots, N ; t \neq n
$$

Based on the analytical ER algorithm in Eq. (A1), we assume

$$
\begin{gathered}
\chi^{1}=\prod_{k=1}^{L}\left(1-w_{k} \sum_{i=1}^{N} \beta_{i, k}\right) \\
\chi^{2}=\prod_{k=1}^{L}\left(1-w_{k}\right)
\end{gathered}
$$

Hence, we can obtain

$$
\begin{gathered}
\beta_{n}=\frac{\beta_{n}^{E R-C}-\chi^{1}}{\sum_{i=1}^{N} \beta_{i}^{E R-C}-(N-1) \chi^{1}-\chi^{2}}> \\
\frac{\beta_{t}^{E R-C}-\chi^{1}}{\sum_{i=1}^{N} \beta_{i}^{E R-C}-(N-1) \chi^{1}-\chi^{2}}=\beta_{t} \\
\Leftrightarrow \beta_{n}^{E R-C}>\beta_{t}^{E R-C} ; t=1, \ldots, N ; t \neq n
\end{gathered}
$$

It follows that the ER-C algorithm is the same as the analytical ER algorithm in EBRBS for classification.

Example 2. Suppose there are two EBRs and their belief distributions of the consequent attribute are shown as follows:

$$
\begin{gathered}
R_{k}:\left\{\left(D_{n}, \beta_{n, k}\right) ; n=1,2\right\} \text { with } \sum_{n=1}^{2} \beta_{n, k}=1 \\
R_{l}:\left\{\left(D_{n}, \beta_{n, l}\right) ; n=1,2\right\} \text { with } \sum_{n=1}^{2} \beta_{n, l}=1
\end{gathered}
$$

The activation weight of the rule $R_{k}$ and $R_{l}$ are assumed to be 
$w_{k}$ and $w_{l}$, respectively, and $w_{k}+w_{l}=1$.

According to the analytical ER algorithm in Eq. (A1) and the ER-C algorithm in Eq. (5), the difference of the integrated belief degrees for the $D_{1}$ and $D_{2}$ can be expressed as follows:

$$
\begin{gathered}
d^{E R}=\beta_{1}-\beta_{2} \\
d^{E R-C}=\beta_{1}^{E R-C}-\beta_{2}^{E R-C}
\end{gathered}
$$

To illustrate the possible values of $d^{E R}$ and $d^{E R-C}$, we consider nine illustrative cases under the assumptions that $\beta_{1, l}>\beta_{1, k}, \beta_{1, l}=$ $\beta_{1, k}$, and $\beta_{1, l}<\beta_{1, k}$ while the value of $w_{k}$ lies between 0 and 1 . Without loss of generality, the value of both $\beta_{1, l}$ and $\beta_{1, k}$ is assumed as $0.3,0.5$, and 0.7 , respectively. Hence, the curves of $d^{E R}$ and $d^{E R-C}$ are shown in Fig. 7. Fig. 7 shows that $d^{E R}$ and $d^{E R-C}$ have same negative and positive symbols for nine combinations based on different belief degrees and activation weights. Hence, the ER-C algorithm can produce the same estimated class as the ER algorithm.

Remark 3. From Theorem 2, the ER-C algorithm can be used to replace the ER algorithm in the inference scheme of the EBRBS while facing classification problems. Additionally, the existing studies of using the ER algorithm as inference engine for classification problems, such as [6] and [7], can also use the ER-C algorithm to replace the inference engine.

Remark 4. The advantages of the ER-C algorithm over the ER algorithm can be summarized as follows:

(1) The ER-C algorithm has a much clean and simple formula than the ER algorithm because it derives from the core part of the ER algorithm.

(2) The ER-C algorithm is more efficient than the ER algorithm according to their time complexity, in which the ERC algorithm is $O(L \times N)$ and the ER algorithm is $O\left(L \times N^{2}\right)$.

(3) In term of independence, the calculation of the integrated belief degree for all classes is independent of each other in the ER-C algorithm so that it is possible to have more solutions of parallelization for the EBRBS.

\section{B. Domain division-based rule reduction method for EBRBS}

In order to further reduce the time complexity of the EBRBS, in this subsection, a domain division-based rule reduction method is proposed for the rule generation scheme. Firstly, the main idea, which follows the similar way to the fuzzy partition [17] and the Wang-Mendel model [31], of the proposed rule reduction method are given based on the following definitions.

Definition 1 (Division point). The division point is the intersection between transform functions used to calculate the belief degree to which the input data belongs to the reference value of antecedent attributes. For convenience, $P\left(A_{i, j}, A_{i, j+1}\right)$ $\left(i=1, \ldots, M ; j=1, \ldots, J_{i}-1\right)$ is defined to express the division point between the $A_{i, j}$ and $A_{i, j+1}$ in the $i$ th antecedent attribute.

Definition 2 (Division domain). The division domain is the local input space constructed by the two adjacent division points of each antecedent attribute. For convenience, $D\left(A_{1, j_{1}}, \ldots, A_{M, j_{M}}\right)\left(j_{i}=1, \ldots, J_{i} ; i=1, \ldots, M\right)$ is defined to express the division domain constructed by the division point regarding the reference values $A_{1, j_{1}}, \ldots, A_{M, j_{M}}$.

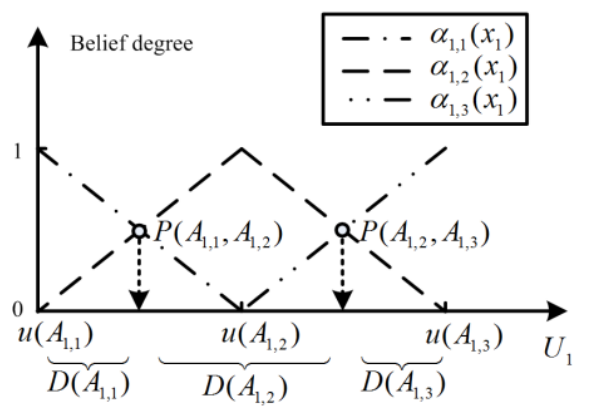

Fig. 8. Example of division point and division domain

Example 3. Suppose that an EBRB includes one antecedent attribute $U_{1}$ with three reference values $A_{1, i}$ and their utility values $u\left(A_{1, i}\right)(i=1,2,3)$. Without loss of generality, the order of those utility values is $u\left(A_{1,1}\right)<u\left(A_{1,2}\right)<u\left(A_{1,3}\right) . x_{1}$ is assumed to be the input variable of the attribute $U_{1}$ and the utility function used to generate belief degrees of the reference value $A_{1, i}(i=1$, $2,3)$, is assumed to be piecewise linear [35], namely $\alpha_{1, i}\left(x_{1}\right)$, shown in Fig. 8. From Fig. 8, there are two division points, namely $P\left(A_{1,1}, A_{1,2}\right)$ and $P\left(A_{1,2}, A_{1,3}\right)$, and three division domains, namely $D\left(A_{1,1}\right), D\left(A_{1,2}\right)$, and $D\left(A_{1,3}\right)$.

Remark 5. Based on Definitions 1 and 2 together with Example 3, when an input data falls into a division domain, the relatively maximal belief degree can be generated from the input data for all reference values belonging to the division domain. In other words, each division domain can be regarded as the clustering center of the input data which most likely belong to the reference value of division domain in the form of belief distribution.

Definition 3 (Rule clustering strategy). The rule clustering strategy is the map relationship between EBRs and division domains based on the relatively maximal belief degree in each antecedent attribute so that it can be defined as

$$
R_{k} \rightarrow D\left(A_{1, j_{1}}, \ldots, A_{M, j_{M}}\right)
$$

where $j_{i}=\arg \max _{j=1, \ldots, J_{i}}\left\{\alpha_{i, j}^{k}\right\}(k=1, \ldots, L ; i=1, \ldots, M) ; \alpha_{i, j}^{k}$ denotes the belief degree of the reference value $A_{i, j}$ in the $k$ th EBR $\left(j=1, \ldots, J_{i} ; i=1, \ldots, M\right), M$ is the number of antecedent attributes, $L$ is the number of EBRs, and $J_{i}$ is the number of reference values used for the $i$ th antecedent attribute.

Definition 4 (Rule reduction strategy). The rule reduction strategy is the combination strategy for the EBRs which are assigned to the same division domain so that it can be defined as

$$
\bar{\alpha}_{i, j}^{l}=\frac{\sum_{k=1}^{L_{l}} \alpha_{i, j}^{k}}{L_{l}}, \bar{\beta}_{n, l}=\frac{\sum_{k=1}^{L_{l}} \beta_{n, k}}{L} ; \text { while } L_{l}>0
$$

where $L_{l}$ is the number of the rules gathered at the $l$ th $(l=1, \ldots$, $\left.\prod_{i=1}^{M} J_{i}\right)$ division domain and $\beta_{n, k}(n=1, \ldots, N ; k=1, \ldots, L)$ denote the belief degrees of the reference value $A_{i, j}$ and $D_{n}$ in the $k$ th rule, respectively.

Remark 6. As shown in Eq. (15), different denominators are 


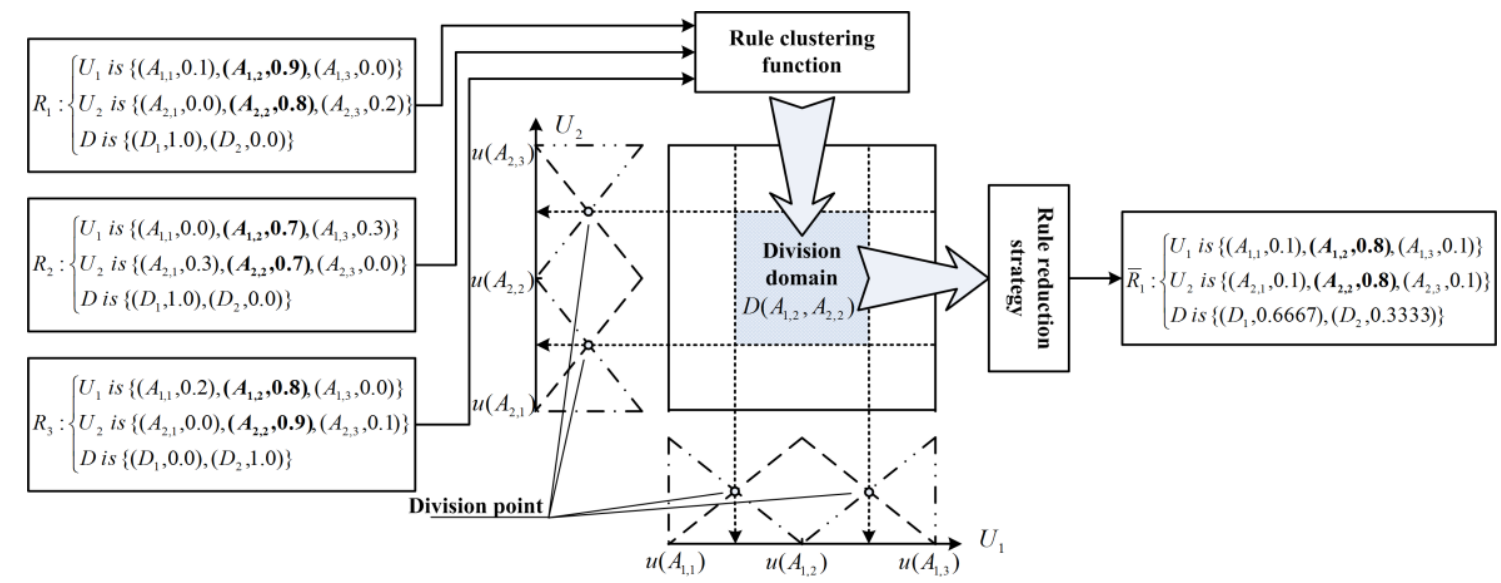

Fig. 9. Examples of rule clustering strategy and rule reduction strategy

used to calculate the belief degree of antecedent and consequent attributes. The main reason is that: (1) the belief degree of antecedent attribute reflects the space relationship between different division domains in a sense, so the belief degree is calculated by using $L_{l}$; (2) the belief degree of consequent attribute reflects how many rules have the same class, thus the belief degree is calculated by using $L$.

Example 4. Suppose that an EBRB includes two antecedent attributes $U_{1}$ and $U_{2}$ with three reference values $A_{i, j}$ and their utility values $u\left(A_{i, j}\right) \quad(i=1,2 ; j=1,2,3)$. Without loss of generality, the order of those utility values is $u\left(A_{i, 1}\right)<u\left(A_{i, 2}\right)$ $<u\left(A_{i, 3}\right)$ in the $i$ th antecedent attribute. In addition, the EBRB has three EBRs $R_{1}, R_{2}$, and $R_{3}$, respectively, as shown in Fig. 9.

From Fig. 9, four division points and nine division domains can be constructed based on Definitions 1 and 2. After that, according to Definition 3, all three EBRs are assigned to the division domain $D\left(A_{1,2}, A_{2,2}\right)$. Afterwards, based on Definition 4 , the EBRs gathered in $D\left(A_{1,2}, A_{2,2}\right)$ are all combined as a new rule, denoted as $\bar{R}_{1}$, which is obtained via Eq. (15), e.g., $\bar{\alpha}_{1,1}^{1}$ $=(0.1+0+0.2) / 3=0.1, \bar{\alpha}_{1,2}^{1}=(0.9+0.7+0.8) / 3=0.8$, and $\bar{\alpha}_{1,3}^{1}=(0$ $+0.3+0) / 3=0.1$ in the antecedent attribute $U_{1}$, and $\bar{\beta}_{1,1}=(1+1+$ $0) / 3=0.6667$ and $\bar{\beta}_{2,1}=(0+0+1) / 3=0.3333$ in the attribute $D$.

Remark 7. Based on Definitions 3 and 4 together with Example 4, when one more EBR is assigned to the same division domain, a new EBR would be generated, in which the information of the new EBR is considered complete because it is generated by using the belief distribution of the antecedent and consequent attribute of all original rules.

Based on the above Definitions 1 to 4 and Examples 3 to 4, the steps of the domain division-based rule reduction method are described as follows:

Step 1: To generate division points for each antecedent attribute by using the transform functions. Suppose that there are $M$ antecedent attributes with $J_{i}$ reference values for the $i$ th $(i=1, \ldots, M)$ antecedent attribute. Based on Definition $1, J_{i}-1$ division points, namely $\left\{P\left(A_{i, j}, A_{i, j+1}\right) ; j=1, \ldots, J_{i^{-}} 1\right\}$, are generated for the $i$ th antecedent attribute.

Step 2: To generate division domains for the EBRBS by using the division points. Based on Definition 2, $\prod_{i=1}^{M} J_{i}$ division domains, namely $\left\{D\left(A_{1, j_{1}}, \ldots, A_{M, j_{M}}\right) ; j_{i}=1, \ldots, J_{i} ; i=\right.$ $1, \ldots, M\}$, are generated for the EBRBS, in which the division domain is the clustering center according to Remark 5.

Step 3: To assign all EBRs to the division domains based on the rule clustering strategy. Suppose that there are $T$ EBRs transformed from $T$ sample data. Based on Definition 3, these $T$ EBRs are all assigned to the $\prod_{i=1}^{M} J_{i}$ division domains.

Step 4: To generate new EBRs from the EBRs gathered in each division domain based on the rule reduction strategy. Suppose that there are $\bar{L}$ division domains which include at least one EBR, based on Definition 4, all EBRs in each division domain are used to generate $\bar{L}$ new EBR, respectively.

Remark 8. After utilizing the domain division-based rule reduction method to downsize the EBRB, $\bar{L}$ new EBRs shown in Step 4 can construct a reduced EBRB, in which the number of rules regarding the reduced EBRB should be no more than both the number of sample data $T$ and the number of division domains $\prod_{i=1}^{M} J_{i}$.

C. Micro-EBRBS: EBRBS with rule reduction and ER-C algorithm but without rule weight calculation

Based on the above analysis and new improvements, a novel EBRBS, called Micro-EBRBS which has a simplified rule generation and inference schemes and a downsized EBRB comparing to EBRBS, is developed and its methodological framework is shown in Fig. 10.

It is clear from Fig. 10 that, comparing to the EBRBS shown in Figs. 1 and 2, the Micro-EBRBS includes the process of rule reduction but exclude the process of rule weight calculation. Additionally, the ER-C algorithm is used to replace the ER algorithm in the process of activated rule integration. More specifically, the rule generation and the inference scheme of the Micro-EBRBS are described as follows.

For the rule generation scheme of the Micro-EBRBS, it consists of the following two steps:

Step 1: Generation of belief distributions using the transformation technique. 


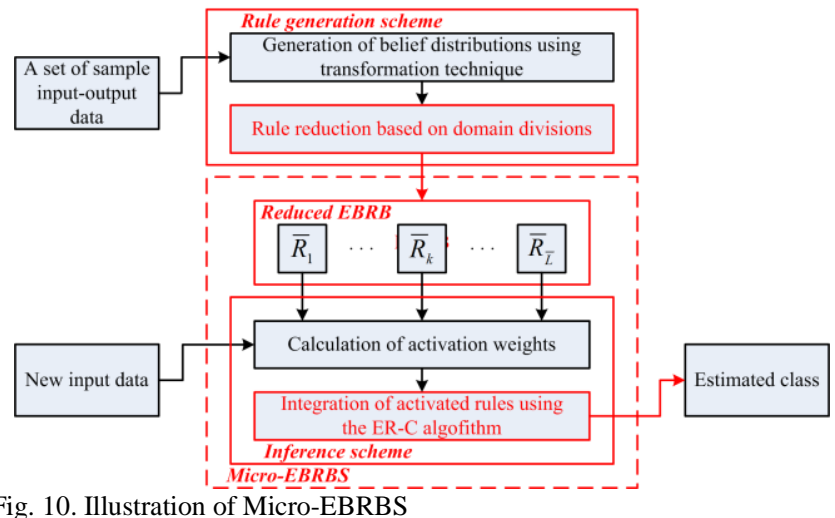

Fig. 10. Illustration of Micro-EBRBS

After determining the basic parameters based on expert knowledge, including attribute weights, utility values of the reference values used for antecedent and consequent attributes, the belief distributions of the antecedent and consequent attributes can be generated.

Suppose that $\left\{u\left(A_{i, j}\right) ; j=1, \ldots, J_{i}\right\}$ is a set of given utility values used for the $i$ th $(i=1, \ldots, M)$ antecedent attribute, and $x_{k, i}$ is the $k$ th $(k=1, \ldots, L)$ sample input data of the $i$ th antecedent attribute. Thus, the belief distribution of the $i$ th antecedent attribute generated using the utility-based equivalence transformation technique [35] is:

$$
S\left(x_{k, i}\right)=\left\{\left(A_{i, j}, \alpha_{i, j}^{k}\right) ; j=1, \ldots, J_{i}\right\}
$$

where

$$
\begin{aligned}
& \alpha_{i, j}^{k}=\frac{u\left(A_{i, j+1}\right)-x_{k, i}}{u\left(A_{i, j+1}\right)-u\left(A_{i, j}\right)} \\
& \alpha_{i, j+1}^{k}=1-\alpha_{i, j}^{k}, \text { if } u\left(A_{i, j}\right) \leq x_{k, i} \leq u\left(A_{i, j+1}\right) \\
& \alpha_{i, t}^{k}=0, \text { for } t=1, \ldots, J_{i} \text { and } t \neq j, j+1
\end{aligned}
$$

where $\alpha_{i, j}^{k}$ is the belief degree of $A_{i, j}$ in the $k$ th EBR obtained from the sample input data $x_{k, i}$.

Next, when the $k$ th sample output data is $y_{k}$ and the given utility values attached to the consequent attribute $D$ are $\left\{u\left(D_{n}\right)\right.$; $n=1, \ldots, N\}$, the belief distribution of consequent attribute is:

$$
S\left(y_{k}\right)=\left\{\left(D_{n}, \beta_{n}^{k}\right) ; n=1, \ldots, N\right\}
$$

Finally, all belief distributions shown in Eqs (16) and (19) together with attribute weights determined by experts are used to construct an EBRB.

Step 2: Rule reduction based on domain divisions.

For the EBRB generated by Step 1, the domain divisionbased rule reduction method shown in Section III-B is then used to reduce the number of rules. Suppose there are $M$ antecedent attributes with $J_{i}$ reference values $A_{i, j}\left(j=1, \ldots, J_{i}\right)$ and one consequent attribute $D$ with $N$ classes $D_{n}(n=1, \ldots, N)$, the $k$ th $(k=1, \ldots, \bar{L})$ rule of the reduced EBRB can be written as

$$
\begin{aligned}
\bar{R}_{k} & \text { IF } U_{1} \text { is }\left\{\left(A_{1, j}, \bar{\alpha}_{1, j}^{k}\right) ; j=1, \ldots, J_{1}\right\} \wedge \cdots \wedge U_{M} \text { is } \\
& \left\{\left(A_{M, j}, \bar{\alpha}_{M, j}^{k}\right) ; j=1, \ldots, J_{M}\right\}, \text { THEN } D \text { is } \\
& \left\{\left(D_{n}, \bar{\beta}_{n, k}\right) ; n=1, \ldots, N\right\} ; \text { with } \theta_{k}=1 \text { and }\left\{\delta_{1}, \ldots, \delta_{M}\right\}
\end{aligned}
$$

where $\bar{\alpha}_{i, j}^{k}$ and $\bar{\beta}_{n, k}$ are the integrated belief degree of the $A_{i, j}$ and the class $D_{n}$ using the rule reduction strategy.

Remark 9. As shown in the proposed rule reduction method, the most complex step is to generate $\bar{\alpha}_{i, j}^{k}(k=1, \ldots, \bar{L} ; i=1, \ldots$, $\left.M ; j=1, \ldots, J_{i}\right)$ and $\bar{\beta}_{n, k}(n=1, \ldots, N)$ by using $L$ rules of EBRB. Furthermore, Section II-C shows that the time complexity of generating belief distributions is $O\left(L \times\left(\sum_{i=1, \ldots, M} J_{i}+N\right)\right)$. Hence, the time complexity for the rule generation scheme of the Micro-EBRBS is $O\left(L \times \bar{L} \times\left(\sum_{i=1, \ldots, M} J_{i}+N\right)\right)$.

For the inference scheme of the Micro-EBRBS, it consists of the following two steps:

Step 1: Calculation of activation weights using the distance measure.

While a test input data is provided for the Micro-EBRBS, the activation weights can be calculated for each EBR of the reduced EBRB. Suppose that $\boldsymbol{x}=\left(x_{1}, \ldots, x_{M}\right)$ is a test input data, each input $x_{i}(i=1, \ldots, M)$ will be firstly transformed into a belief distribution of the reference values of the $i$ th antecedent attribute using Eqs. (17) and (18).

$$
S\left(x_{i}\right)=\left\{\left(A_{i, j}, \alpha_{i, j}\right) ; j=1, \ldots, J_{i}\right\}
$$

Next, the individual matching degree of the $i$ th antecedent attribute in the $k$ th rule, denoted as $S^{k}\left(x_{i}, U_{i}\right)$, is calculated by using the Euclidean distance:

$$
\begin{aligned}
S^{k}\left(x_{i}, U_{i}\right) & =1-d^{k}\left(x_{i}, U_{i}\right) \\
& =1-\min \left\{1, \sqrt{\sum_{j=1}^{J i}\left(\alpha_{i, j}-\alpha_{i, j}^{k}\right)^{2}}\right\}
\end{aligned}
$$

where $d^{k}\left(x_{i}, U_{i}\right)$ is the distance measurement.

Finally, the activation weight of the $k$ th EBR, denoted as $w_{k}$, is calculated by

$$
w_{k}=\frac{\theta_{k} \prod_{i=1}^{M}\left(S^{k}\left(x_{i}, U_{i}\right)\right)^{\bar{\delta}_{i}}}{\sum_{l=1}^{L}\left(\theta_{l} \prod_{i=1}^{M}\left(S^{l}\left(x_{i}, U_{i}\right)\right)^{\bar{s}_{i}}\right)}, \bar{\delta}_{i}=\frac{\delta_{i}}{\max _{i=1, \ldots, M}\left\{\delta_{i}\right\}}
$$

where $\theta_{k}$ is the weight of the $k$ th rule; $\delta_{i}$ is the weight of the $i$ th antecedent attribute.

Step 2: Integration of activated rules using the ER-C algorithm.

After performing Step 1, all activated rules can be integrated using the ER-C algorithm shown in Eq. (5) and the integrated belief distribution of the test input data $\boldsymbol{x}$ can be represented as follows:

$$
f(\boldsymbol{x})=\left\{\left(D_{n}, \beta_{n}^{E R-C}\right) ; n=1, \ldots, N\right\}
$$

Afterwards, the estimated class for the test input data $\boldsymbol{x}$ can be obtained as follows:

$$
f(\boldsymbol{x})=D_{n}, n=\arg \max _{i=1, \ldots N}\left\{\beta_{i}^{E R-C}\right\}
$$

Remark 10. Considering that the inference scheme of the Micro-EBRBS is based on the reduced EBRB, which only has $\bar{L}$ rules, the time complexity of the inference scheme 


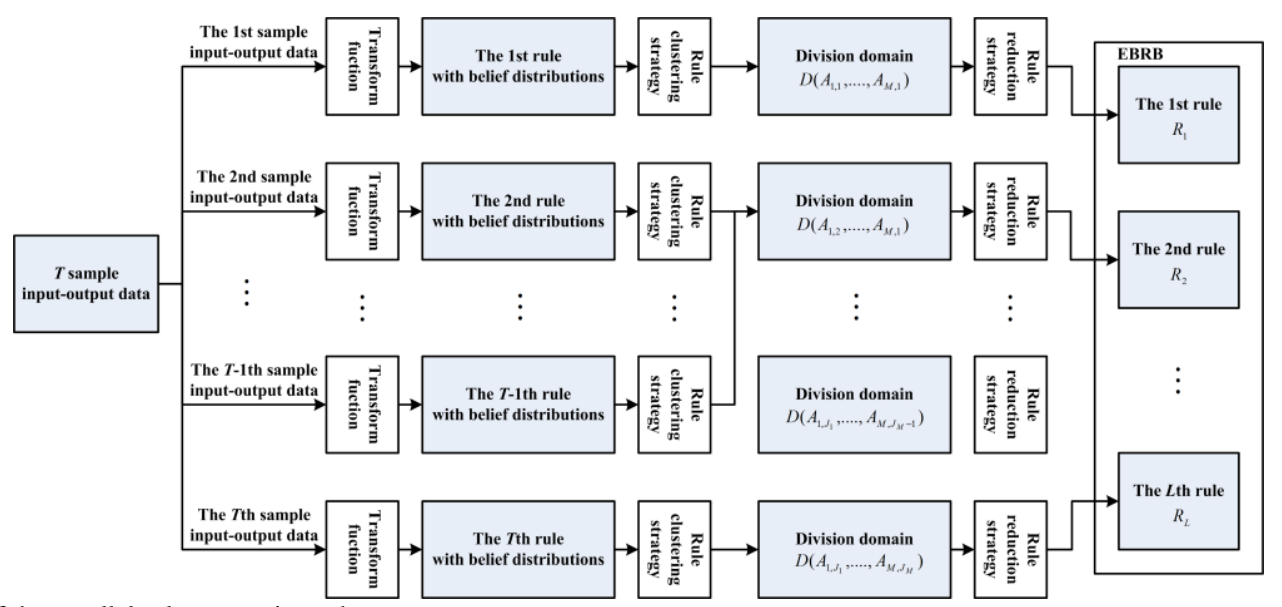

Fig. 11. Illustration of the parallel rule generation scheme

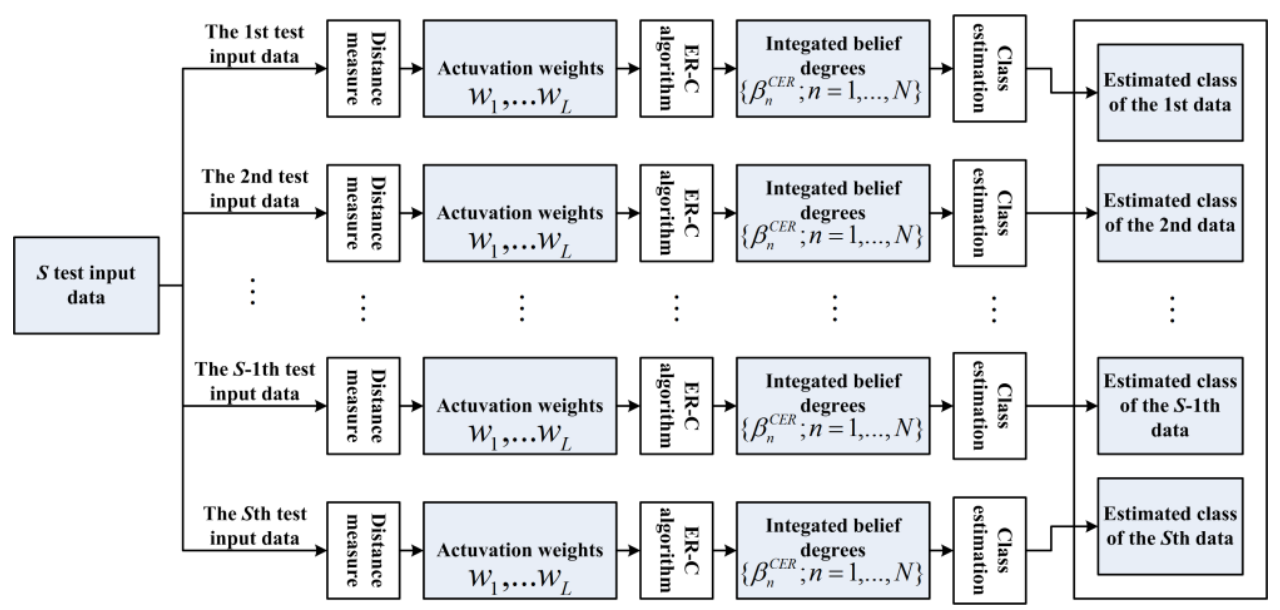

Fig. 12. Illustration of the parallel inference scheme

regarding the Micro-EBRBS is $O\left(\bar{L} \times\left(\sum_{i=1, \ldots,}{ }_{M} J_{i}+N\right)\right)$ for classifying each test data.

D. Apache Spark-based implementation of Micro-EBRBS for big data multi-class classification

In order to further improve the computing efficiency of the Micro-EBRBS, the Apache Spark is used to achieve the parallel rule generation and inference schemes. As an in-memory big data platform, the Apache Spark has been proven that it supports a much wider range of functionality than the Apache Hadoop [9]. The fundamental data structure of the Apache Spark is the resilient distributed dataset (RDD), which represents a collection of distributed items that can be manipulated across many computing nodes concurrently. Hence, the RDD allows the data cache to be stored in memory and perform computations for the same data directly from memory. After the RDD is constructed, the program can perform the following two operations:

(1) Transformations: this kind of operation is to create a new RDD from existing RDD and the concrete function includes map (which is to return a new RDD formed by passing each element of the source through a function), mapToPair (which is to return a new RDD of key-value pairs by using a function), reduceByKey (which is to return a new RDD of key-value pairs where the values for each key are aggregated using the given reduce function), and so on. The detailed description of those functions can be found in [4].

(2) Actions: this kind of operation is to return the final results of RDD computations and the concrete function includes reduce (which is to aggregate the elements of the RDD using a function), collect (which is to return all the elements of the RDD as an array at the driver program), and others. The detailed description of those functions can be found in [4].

Based on the functions of transformation and action, the pseudocodes are provided to illustrate the Apache Spark-based implementation of the parallel rule generation and inference schemes of Micro-EBRBS below respectively.

\section{EXPERIMENTS}

The performance of the Micro-EBRBS is empirically assessed through three different experiments with 14 classification datasets from the well-known UCI repository of machine learning databases [30]. The EBRBS, the conventional FRBCS and machine-learning classifiers, and the big data FRBCS classifiers are used to compare in terms of the accuracy and computing efficiency, respectively.

\section{A. Datasets and experiment conditions}

Fourteen classification datasets obtained from UCI are used to evaluate the performance of the Micro-EBRBS. The main 


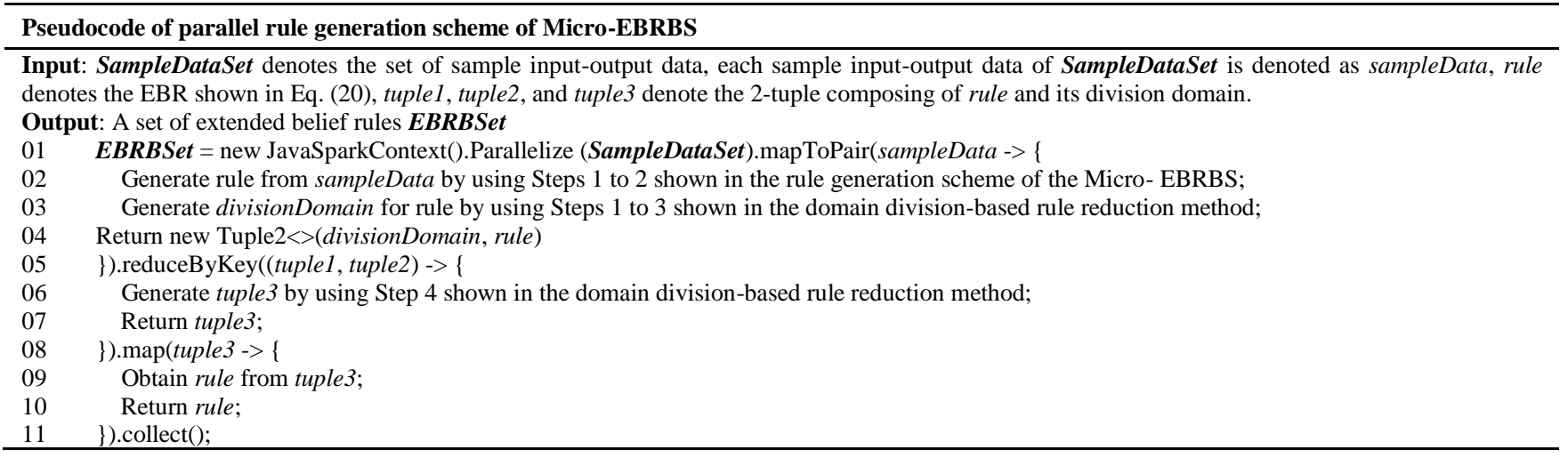

\section{Pseudocode of parallel inference scheme of Micro-EBRBS}

Input: TestDataSet denotes the set of test input data, each test input data of TestDataSet is denoted as testData, classl denotes the estimated class of the Micro-EBRBS, and class2 denotes the actual class of test input data, $a$ and $b$ denote the integer variable.

Output: The total number of test input data correctly classified by the Micro-EBRBS totalCorrect.

01 totalCorrect $=$ new JavaSparkContext().parallelize $($ TestDataSet $)$

$02 \quad . m a p(t e s t$ Data $->\{$

03 Generate class 1 for testData by using Steps 1 to 2 shown in the inference scheme of the Micro-EBRBS;

04 Obtain class2 from testData;

$05 \quad$ Return class $1==$ class $2 ? 1: 0$;

$06 \quad 3)$.reduce $((a, b)->a+b)$;

TABLE I

STATISTICS ON FOURTEEN CLASSIFICATION DATASETS

\begin{tabular}{ccccc}
\hline \hline No. & Dataset & No. of data & No. of attributes & No. of classes \\
\hline 1 & Diabetes & 393 & 8 & 2 \\
2 & Cancer & 569 & 30 & 2 \\
3 & Transfusion & 748 & 4 & 2 \\
4 & Banknote & 1,372 & 4 & 2 \\
5 & Magic & 19,020 & 10 & 2 \\
6 & Wine & 178 & 13 & 3 \\
7 & Waveform & 5,000 & 21 & 3 \\
8 & Glass & 214 & 9 & 6 \\
9 & Red wine & 1,599 & 11 & 6 \\
10 & Satimage & 6,435 & 36 & 6 \\
11 & Census & 95,130 & 40 & 2 \\
12 & Gas sensors & 928,991 & 10 & 3 \\
13 & Covtype & 581,012 & 54 & 7 \\
14 & Poker & $1,025,010$ & 10 & 10 \\
\hline \hline
\end{tabular}

characteristics of these datasets are summarized in Table I. Notice that for the datasets Diabetes, Cancer, and Census, we have removed the data with missing attribute values.

To develop the comparison in multiple aspects, $k$-fold crossvalidation $(\mathrm{K}-\mathrm{CV})$ is considered in the experiments, where each dataset is divided into $k$ blocks, with $k-1$ blocks as training data, namely sample input-output data, and the remaining block as testing data. Additionally, the nonparametric statistical analysis is used to assess if significant differences exist among different classifiers at a level of significance of $\alpha=0.1$. For conducting multiple statistical comparisons over multiple datasets, as suggested in [14], the Friedman and Holm tests are employed.

For the first and the second experiments (Sections IV-B and IV-C), the datasets with relatively small number of data, including the 1 st to the 10th datasets, are used to compare the performance of the Micro-EBRBS with the EBRBS and the conventional FRBCS and machine-learning classifiers. All these classifiers are implemented using Java programming (JDK 1.8.0) and the open source software (Weka and KEEL) on Intel (R) Core (TM) i5-4300U CPU at $1.90 \mathrm{GHz}$ and 4GB RAM with Windows 7. For each dataset, the average results of the 10 runs of each classifier are used to compare their performances.

For the third experiment (Section IV-D), the datasets with relatively large number of data, including the 11th to the 14th datasets, are used to compare the performance of the MicroEBRBS with the big data FRBCS classifiers, and all these classifiers are executed in the 17 nodes cluster connected via 8GT/s Ethernet LAN network, where the master node is composed of 1 Intel Xeon E5-2640 4 cores at $2.5 \mathrm{GHz}$ and 16GB RAM and the slave nodes are composed of 2 Intel Xeon E5-2670v2 10 cores at $2.5 \mathrm{GHz}$ and 64GB RAM. The entire cluster runs under Red Hat 7.3 and Apache Spark 2.1.0.

\section{B. Comparative analysis between Micro-EBRBS and EBRBS}

The first experiment aims to compare the accuracy and computing efficiency of the Micro-EBRBS with the EBRBS, respectively, and the comparisons are based on the 2/4/6/8/10$\mathrm{CV}$ to investigate the influences on the accuracy and computing efficiency of these EBRBSs by using different numbers of training and testing data.

For the basic parameters of the Micro-EBRBS and EBRBS, suppose that all attribute weights are 1, namely

$$
\delta_{i}=1 ; i=1, \ldots, M
$$

where $M$ is the number of antecedent attributes. The number of reference values is three for each antecedent attribute, and the utility value of these reference values is defined as follows:

$$
\left\{u\left(A_{i, j}\right) ; j=1,2,3\right\}=\left\{l b_{i}, \frac{l b_{i}+u b_{i}}{2}, u b_{i}\right\} ; i=1, \ldots, M
$$

where $u\left(A_{i, j}\right)$ denotes the utility value of the reference value $A_{i, j}$, $l b_{i}$ and $u b_{i}$ denote the lower and upper bounds of the $i$ th antecedent attribute, respectively. 


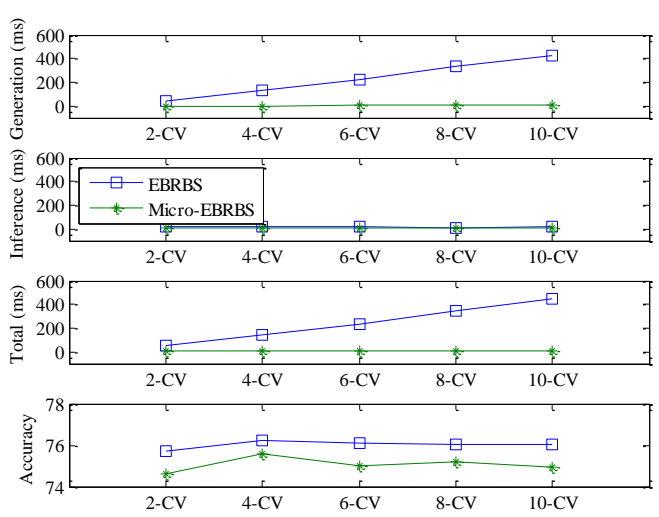

(a) Diabete

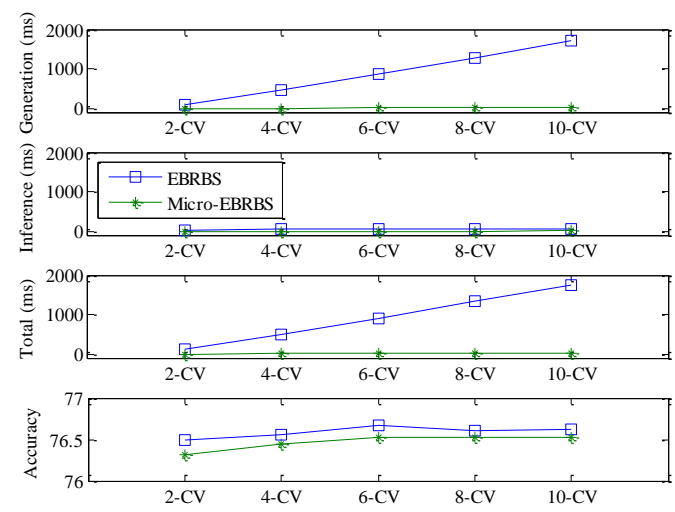

(c) Transfusion
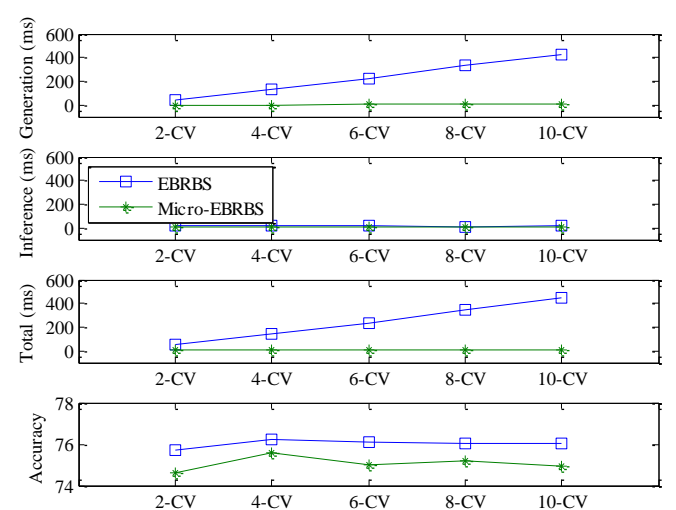

(b) Cancer
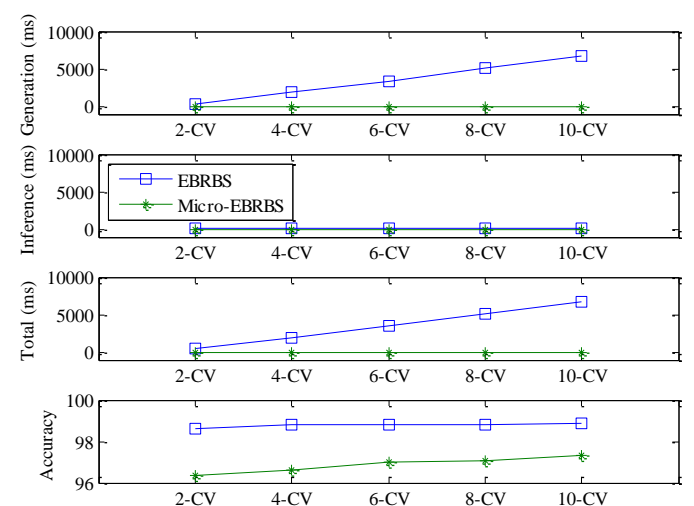

(d) Banknote

Fig. 13. Comparison of computing time and accuracy between Micro-EBRBS and EBRBS for two-class datasets

TABLE II

COMPARISON OF COMPUTING EFFICIENCY FOR MICRO-EBRBS AND EBRBS

\begin{tabular}{|c|c|c|c|c|c|c|c|c|c|c|}
\hline \multirow{2}{*}{$\begin{array}{l}\text { No. of } \\
\text { classes }\end{array}$} & \multirow{2}{*}{ Dataset } & \multicolumn{2}{|c|}{ Rule generation (ms) } & \multirow{2}{*}{ Ratio } & \multicolumn{2}{|c|}{ Inference $(\mathrm{ms})$} & \multirow{2}{*}{ Ratio } & \multicolumn{2}{|c|}{ Total (ms) } & \multirow{2}{*}{ Ratio } \\
\hline & & Micro-EBRBS & EBRBS & & Micro-EBRBS & EBRBS & & Micro-EBRBS & EBRBS & \\
\hline \multirow[t]{4}{*}{ Two-class } & Diabetes & 6.1 & 429.0 & 0.014 & 3.2 & 15.6 & 0.205 & 9.3 & 444.6 & 0.021 \\
\hline & Cancer & 40.8 & $1,475.5$ & 0.028 & 69.9 & 76.7 & 0.911 & 110.7 & $1,552.2$ & 0.071 \\
\hline & Transfusion & 3.2 & $1,709.6$ & 0.002 & 3.0 & 37.5 & 0.080 & 6.2 & $1,747.1$ & 0.004 \\
\hline & Banknote & 12.4 & $6,645.2$ & 0.002 & 3.2 & 148.3 & 0.022 & 15.6 & $6,793.5$ & 0.002 \\
\hline \multirow[t]{4}{*}{ Multi-class } & Wine & 4.6 & 81.1 & 0.058 & 3.2 & 4.7 & 0.681 & 7.8 & 85.8 & 0.091 \\
\hline & Waveform & 750.5 & $205,530.3$ & 0.004 & $3,623.3$ & $8,654.2$ & 0.419 & $4,373.8$ & $214,184.5$ & 0.020 \\
\hline & Glass & 3.0 & 164.4 & 0.018 & 1.1 & 5.3 & 0.208 & 4.1 & 169.7 & 0.024 \\
\hline & Red Wine & 40.7 & $13,291.8$ & 0.003 & 60.2 & 424.9 & 0.142 & 100.9 & $13,716.7$ & 0.007 \\
\hline
\end{tabular}

Figs. 13 and 14 show the computing time and the accuracy regarding the Micro-EBRBS in comparison with the EBRBS over two and multi-class datasets, respectively, in which the computing time includes the time of rule generation scheme, inference scheme and total time.

For the two-class datasets, including Diabetes, Cancer, Transfusion, and Banknote, the computing time of rule generation scheme regarding the EBRBS is increasing with the increasing number of training data used to generate EBRs, e.g., $50 \%$ data are regarded as the training data in $2-\mathrm{CV}$ and $90 \%$ data as the training data in 10-CV. Additionally, there are slight differences between the Micro-EBRBS and the EBRBS in term of the computing time of the inference scheme and the accuracy. As a result, the total computing time of the EBRBS is much more than that of the Micro-EBRBS.
For the multi-class datasets, including Wine, Waveform, Glass, Red wine, the similar conclusions are obtained in terms of the computing time and the accuracy.

In order to show the detailed comparison of the MicroEBRBS and the EBRBS, Tables II and III provide the results of rule generation time, inference time, total time, number of rules, number of activated rules, and the accuracy, in which the ratio is the result of the Micro-EBRBS divided by the result of the EBRBS. Hence, the Micro-EBRBS with a larger ratio in term of accuracy and a smaller ratio in term of computing time is better than the EBRBS.

Table II shows that the computing time of the Micro-EBRBS is much less than that of the EBRBS for all two and multi-class datasets, where the minimum ratio of computing time is obtained from the dataset Banknote and its ratios are 0.002, 


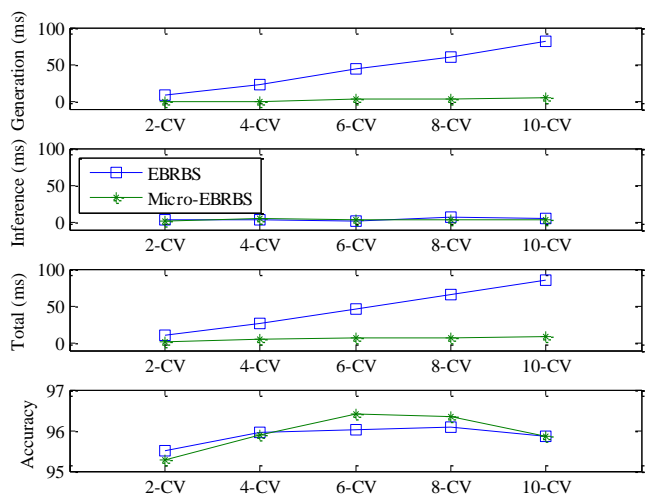

(a) Wine
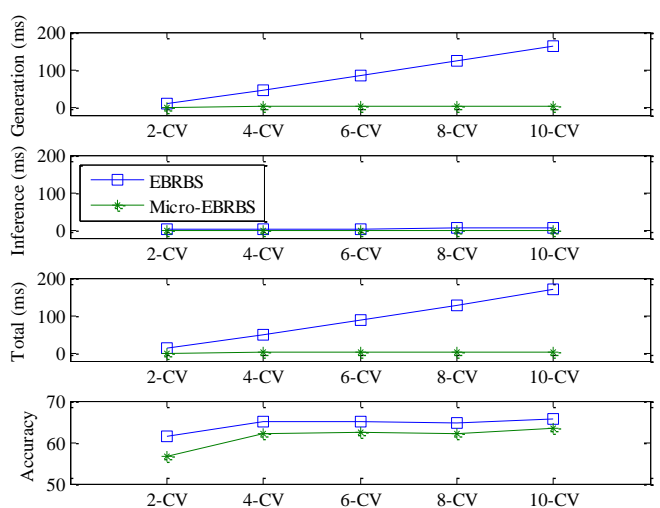

(c) Glass
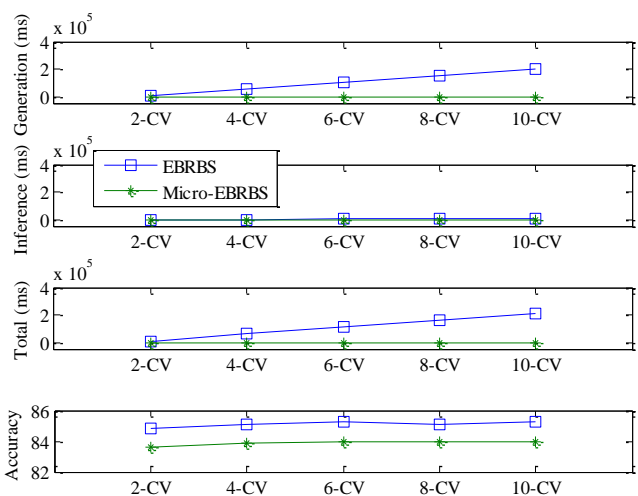

(b) Waveform
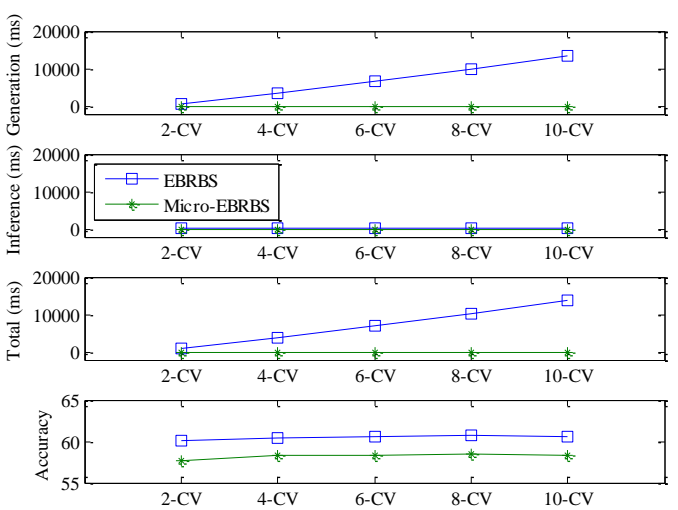

(d) Red wine

Fig. 14. Comparison of computing time and accuracy between Micro-EBRBS and EBRBS for multi-class datasets

TABLE III

COMPARISON OF ACCURACY AND NUMBER RULES AND ACTIVATED RULES FOR MICRO-EBRBS AND EBRBS

\begin{tabular}{|c|c|c|c|c|c|c|c|c|c|c|}
\hline \multirow{2}{*}{$\begin{array}{l}\text { No. of } \\
\text { classes }\end{array}$} & \multirow[b]{2}{*}{ Dataset } & \multicolumn{2}{|c|}{ No. of rules } & \multirow{2}{*}{ Ratio } & \multicolumn{2}{|c|}{ No. of activated rules } & \multirow{2}{*}{ Ratio } & \multicolumn{2}{|c|}{ Accuracy $(\%)$} & \multirow{2}{*}{ Ratio } \\
\hline & & Micro-EBRBS & EBRBS & & Micro-EBRBS & EBRBS & & Micro-EBRBS & EBRBS & \\
\hline \multirow[t]{4}{*}{ Two-class } & Diabetes & 94.0 & 353.7 & 0.266 & 49.6 & 208.9 & 0.237 & 74.91 & 76.01 & 0.986 \\
\hline & Cancer & 448.2 & 512.1 & 0.875 & 222.7 & 256.9 & 0.867 & 96.49 & 96.45 & 1.000 \\
\hline & Transfusion & 12.8 & 673.2 & 0.019 & 7.4 & 533.1 & 0.014 & 76.52 & 76.62 & 0.999 \\
\hline & Banknote & 30.8 & $1,234.8$ & 0.025 & 22.3 & 955.1 & 0.023 & 97.34 & 98.86 & 0.985 \\
\hline \multirow[t]{4}{*}{ Multi-class } & Wine & 126.3 & 160.2 & 0.788 & 45.2 & 58.6 & 0.771 & 95.84 & 95.84 & 1.000 \\
\hline & Waveform & $2,031.7$ & $4,500.0$ & 0.452 & $1,492.9$ & $3,523.1$ & 0.424 & 84.00 & 85.22 & 0.986 \\
\hline & Glass & 41.9 & 192.6 & 0.218 & 11.8 & 95.4 & 0.124 & 63.32 & 65.56 & 0.967 \\
\hline & Red Wine & 230.8 & $1,439.1$ & 0.160 & 139.6 & 964.1 & 0.145 & 58.36 & 60.61 & 0.963 \\
\hline
\end{tabular}

TABLE IV

FRIEDMAN AND HOLM TESTS TO COMPARE THE ACCURACY OF MICRO-EBRBS $\operatorname{AND} \operatorname{EBRB}(\alpha=0.1)$

\begin{tabular}{|c|c|c|}
\hline Indicator & EBRBS (two-class) & EBRBS (Multi-class) \\
\hline $\mathrm{p}$ value & 0.3173 & 0.1336 \\
\hline Critical value & 0.1000 & 0.1000 \\
\hline Hypothesis & Accepted & Accepted \\
\hline
\end{tabular}

0.022 , and 0.002 for the rule generation, inference, and total time, respectively, and the maximum one is obtained from the datasets Cancer and Wine and their ratio are 0.058 and 0.091 for the rule generation time and total time of Wine and 0.911 for the inference time of Cancer.

Table III shows that the number of rules and activated rules involved in the Micro-EBRBS is less than the EBRBS, where the minimum ratio is obtained from the datasets Transfusion, i.e., 0.019 for the number of rules and 0.014 for the number of activated rules, and the maximum one is obtained from the datasets Cancer, 0.875 for the number of rules and 0.867 for the number of activated rules. Furthermore, it is clear from Table III that the accuracy of the Micro-EBRBS closely approximates that of the EBRBS and the range of the ratio can be expressed as $[0.963,1.000]$ for eight classification datasets.

In order to further compare the accuracy of the MicroEBRBS and EBRBS, Friedman and Holm tests are applied to provide the statistical analysis based on two and multi-class datasets, respectively. From Table IV, although the EBRBS can obtain the best accuracy at most of two and multi- class datasets, none of hypotheses is in favor of the significant difference between EBRBS and Micro-EBRBS. However, the computing efficiency of Micro-EBRBS is much better than EBRBS. 
TABLE V

COMPARISON OF ACCURACY FOR MICRO-EBRBS AND FIVE FRBCS CLASSIFIERS

\begin{tabular}{|c|c|c|c|c|c|c|c|}
\hline No. of classes & Dataset & SLAVE & FH-GBML & FURIA & FARC-HD & Chi-FRBCS & Micro-EBRBS \\
\hline \multirow[t]{6}{*}{ Two-class } & Diabetes & $77.10 \%(1.5)$ & $70.23 \%(6)$ & $76.59 \%(3)$ & $77.10 \%(1.5)$ & $72.80 \%(5)$ & $74.91 \%(4)$ \\
\hline & Cancer & $92.33 \%(3)$ & $92.26 \%(5)$ & $90.68 \%(6)$ & $95.25 \%(2)$ & $92.32 \%(2)$ & $96.49 \%(1)$ \\
\hline & Transfusion & $76.60 \%(5)$ & $79.01 \%(1)$ & $78.74 \%(2)$ & $77.27 \%(3)$ & $76.80 \%(3)$ & $76.52 \%(6)$ \\
\hline & Banknote & $91.33 \%(6)$ & $98.18 \%(3)$ & $99.13 \%(2)$ & $99.78 \%(1)$ & $94.42 \%(5)$ & $97.34 \%(4)$ \\
\hline & Magic & $74.96 \%(6)$ & $81.30 \%(3)$ & $84.63 \%(1)$ & $84.51 \%(2)$ & $80.62 \%(2)$ & $77.38 \%(5)$ \\
\hline & Average rank & 4.3 & 3.6 & 2.8 & 1.9 & 4.4 & 4.0 \\
\hline \multirow[t]{6}{*}{ Multi-class } & Wine & $89.47 \%(6)$ & $92.61 \%(3)$ & $91.88 \%(4)$ & $94.35 \%(2)$ & $90.17 \%(5)$ & $95.84 \%(1)$ \\
\hline & Waveform & $81.48 \%$ (4) & $60.18 \%(6)$ & $83.10 \%(3)$ & $83.78 \%(2)$ & $74.70 \%(5)$ & $84.00 \%(1)$ \\
\hline & Glass & $58.05 \%(4)$ & $57.99 \%(5)$ & $58.49 \%(3)$ & $70.24 \%(1)$ & $50.37 \%(6)$ & $63.32 \%(2)$ \\
\hline & Red Wine & $55.60 \%(6)$ & $68.67 \%(1)$ & $57.72 \%(4)$ & $59.72 \%(2)$ & $55.89 \%(5)$ & $58.36 \%(3)$ \\
\hline & Satimage & $81.69 \%(4)$ & $74.72 \%(6)$ & $89.15 \%(1)$ & $87.32 \%(2)$ & $74.79 \%(5)$ & $85.41 \%(3)$ \\
\hline & Average rank & 4.8 & 4.2 & 3.0 & 1.8 & 5.2 & 2.0 \\
\hline
\end{tabular}

TABLE VI

FRIEDMAN AND HOLM TESTS TO COMPARE THE ACCURACY OF MICRO-EBRBS AND FIVE FRBCS CLASSIFIERS $(\alpha=0.1)$

\begin{tabular}{ccccccc}
\hline \hline Item & Indicator & SLAVE & FH-GBML & FURIA & FARC-HD & Chi-FRBCS \\
\hline Two-class & p value & 0.7999 & 0.7353 & 0.3105 & 0.0759 & 0.7353 \\
& Critical value & 0.1000 & 0.0333 & 0.0250 & 0.0200 & 0.0333 \\
& Hypothesis & Accepted & Accepted & Accepted & Accepted & Accepted \\
Multi-class & p value & 0.0180 & 0.0630 & 0.3980 & 0.8658 & 0.0068 \\
& Critical value & 0.0250 & 0.0333 & 0.0500 & 0.1000 & 0.0200 \\
& Hypothesis & Rejected & Accepted & Accepted & Accepted & Rejected \\
\hline \hline
\end{tabular}

TABLE VII

COMPARISON OF COMPUTING TIME IN SECOND FOR MICRO-EBRBS AND FIVE FRBCS CLASSIFIERS

\begin{tabular}{|c|c|c|c|c|c|c|c|}
\hline No. of classes & Dataset & SLAVE & FH-GBML & FURIA & FARC-HD & Chi-FRBCS & Micro-EBRBS \\
\hline \multirow[t]{6}{*}{ Two-class } & Diabetes & $569 \mathrm{~s}(5)$ & $2 \mathrm{~s} 316 \mathrm{~s}(6)$ & $4.3 \mathrm{~s} \mathrm{(3)}$ & $56 \mathrm{~s}(4)$ & 0.0 s (1.5) & 0.0 s (1.5) \\
\hline & Cancer & $1200 \mathrm{~s}(5)$ & $4 \mathrm{~s} 947 \mathrm{~s}(6)$ & $6.8 \mathrm{~s} \mathrm{(3)}$ & $249 \mathrm{~s}(4)$ & 0.1 s (1.5) & 0.1 s (1.5) \\
\hline & Transfusion & $212 \mathrm{~s}(5)$ & 2 s899s (6) & $4.2 \mathrm{~s}(3)$ & $31 \mathrm{~s}(4)$ & $0.0 \mathrm{~s}(1.5)$ & $0.0 \mathrm{~s}(1.5)$ \\
\hline & Banknote & 502 s (5) & $6 \mathrm{~s} 256 \mathrm{~s}(6)$ & $6.0 \mathrm{~s}(3)$ & $69 \mathrm{~s}(4)$ & $0.0 \mathrm{~s}(1.5)$ & $0.0 \mathrm{~s}(\mathbf{1 . 5})$ \\
\hline & Magic & 31,303 s (5) & $387,010 \mathrm{~s}(6)$ & $715 \mathrm{~s}(3)$ & $8,655 \mathrm{~s}(4)$ & $1.0 \mathrm{~s}(1)$ & $1.1 \mathrm{~s}(2)$ \\
\hline & Average rank & 5 & 6 & 3 & 4 & 1.4 & 1.6 \\
\hline \multirow[t]{6}{*}{ Multi-class } & Wine & $268 \mathrm{~s}(5)$ & $1,394 \mathrm{~s}(6)$ & $2.4 \mathrm{~s}(3)$ & $75 \mathrm{~s}(4)$ & $0.0 \mathrm{~s}(1.5)$ & $0.0 \mathrm{~s}(\mathbf{1 . 5})$ \\
\hline & Waveform & $73,570 \mathrm{~s}(6)$ & $45,255 \mathrm{~s}(5)$ & $184 \mathrm{~s}(3)$ & $2,490 \mathrm{~s}(4)$ & $6.5 \mathrm{~s}(2)$ & $4.0 \mathrm{~s}(1)$ \\
\hline & Glass & $611 \mathrm{~s}(5)$ & $1,184 \mathrm{~s}(6)$ & $3.2 \mathrm{~s} \mathrm{(3)}$ & $100 \mathrm{~s}(4)$ & $0.0 \mathrm{~s}(\mathbf{1 . 5})$ & $0.0 \mathrm{~s}(\mathbf{1 . 5})$ \\
\hline & Red Wine & $1,049 \mathrm{~s}(5)$ & $9,434 \mathrm{~s}(6)$ & $21 \mathrm{~s}(3)$ & $590 \mathrm{~s}(4)$ & $0.6 \mathrm{~s}(2)$ & 0.1 s (1) \\
\hline & Satimage & 29,655 s (5) & $62,579 \mathrm{~s}(6)$ & 346 s (3) & 8,876 s (4) & 87 s (2) & $12 \mathrm{~s}(1)$ \\
\hline & Average rank & 5.2 & 5.8 & 3 & 4 & 1.8 & 1.2 \\
\hline
\end{tabular}

In summary, for the comparison of the Micro-EBRBS and EBRBS, the experiment results have shown that the former has much less computing time than the latter. Moreover, the MicroEBRBS is comparable to the EBRBS in term of accuracy while much less number of rules and activated rules are used to address many classification datasets.

\section{Comparative analysis between Micro-EBRBS and conventional classifiers}

The second experiment aims to compare the accuracy of the Micro-EBRBS with the conventional classifiers, which include the FRBCS and the conventional machine-learning classifiers. Apart from the setting of the Micro-EBRBS introduced in Eqs. (26) and (27), the other classifiers are shown as follows:

(1) Chi-FRBCS [29]: it was proposed by Chi et al., where the Penalized Certainty Factor (PCF) is used to calculate rule weights, the winner rule strategy is used as the fuzzy reasoning method, and the OVO is used to improve the performance of Chi-FRBCS in dealing with multi-class classification problems. Here, assume that the number of fuzzy labels is three for each attribute and these fuzzy labels modeled as triangular membership function.
(2) Other FRBCS classifiers: structural learning algorithm on vague environment (SLAVE), fuzzy hybrid genetic-based machine learning algorithm (FH-GBML), fuzzy unordered rule induction algorithm (FURIA), and fuzzy association rule-based classification method for high-dimensional problems (FARCHD), they are all obtained from KEEL software. The setting of these FRBCS classifiers follows the previous study in [3].

(3) Machine-learning classifiers: $k$ nearest neighbor (KNN), Naïve Bayes (NB), Decision Tree (DT), Random Forest (RF), Artificial Neural Network (ANN), and Support Vector Machine (SVM), are all obtained from WEKA software. Apart from the default setting, $20 \%$ number of training data is set as neighbors for the KNN, 5\% number of training data is set as the minimum number of data per leaf for the DT, the number of random trees is set as 5 for the RF, and the number of iterations is set as 10 for the ANN.

Table $\mathrm{V}$ shows the accuracy of Micro-EBRBS in comparison with five FRBCS classifiers, including SLAVE, FH-GBML, FURIA, FARC-HD, and Chi-FRBCS, over two and multi-class datasets, respectively, where the result of the best accuracy is highlighted in bold-face and the number in brackets denotes the rank of each classifier. For the two-class datasets, the accuracy 

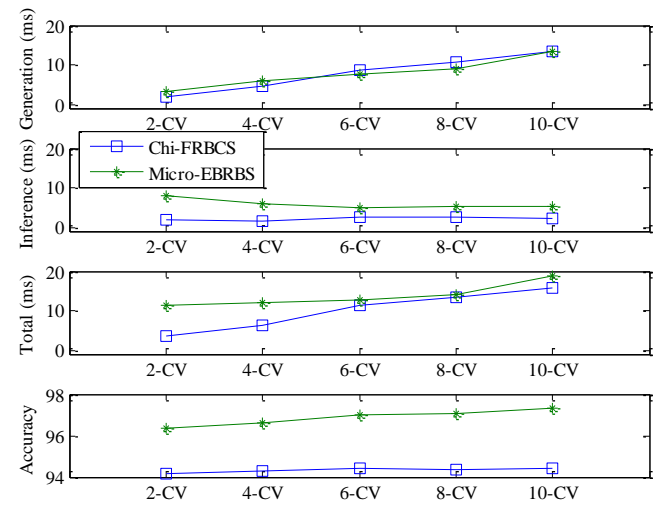

(a) Banknote
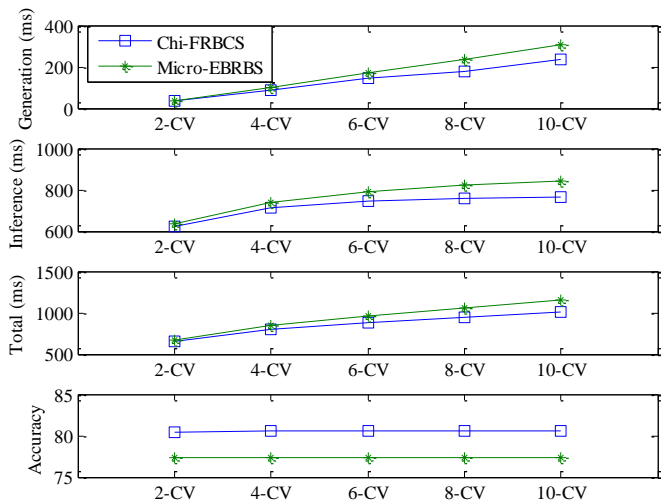

(b) Magic

Fig. 15. Comparison of computing efficiency and accuracy between Chi-FRBCS and Micro-EBRBS for two-class datasets
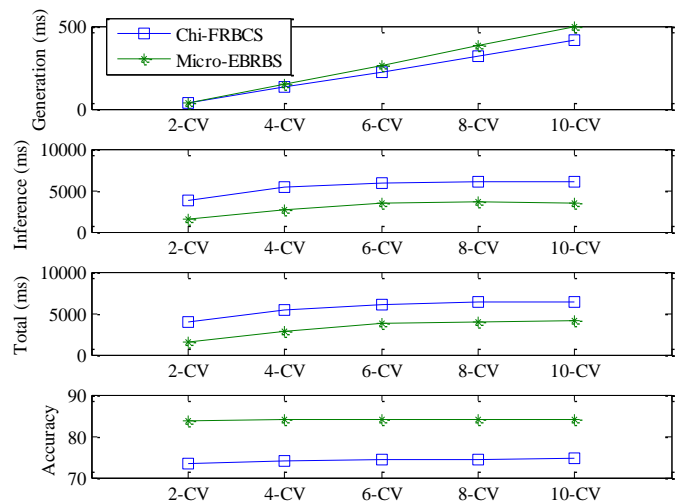

(a) Waveform
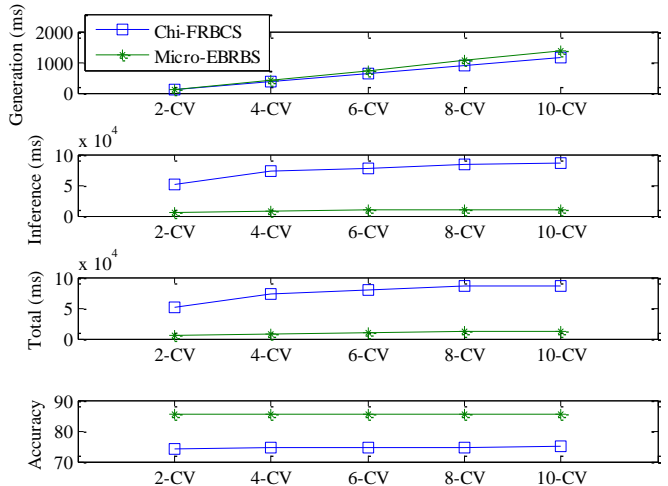

(b) Satimage

Fig. 16. Comparison of computing efficiency and accuracy between Chi-FRBCS and Micro-EBRBS for multi-class datasets

of FARC-HD is better than other FRBCS classifiers as well as Micro-EBRBS ranked at the 3rd place in term of average rank. For the multi-class datasets, the FARC-HD remains its advantages in dealing with classification problems over other classifiers. Despite the fact that the FARC-HD outperforms the Micro-EBRBS, it is still possible to see a considerable decrease in the average rank of Micro-EBRBS, namely from 4.0 to 2.0. This is so because the distributed belief degree is used in each EBR to express multiple classes and Micro-EBRBS therefore has excellent abilities to deal with multi-class problems.

In addition to the accuracy and average rank for each dataset and each classifier shown in Table V, Table VI provides the statistical analysis of accuracy while the Micro-EBRBS is selected as the control method for the Friedman and Holm tests. As shown in Table VI, apart from the SLAVE and Chi-FRBCS in the case of multi-class datasets, all hypotheses regarding five FRBCS classifiers are accepted, which means that although some of FRBCS classifiers, such as the FARCH-HD, FURIA, and FH-GBML, are better than Micro-EBRBS, none of hypotheses is in favor of the significant differences between the FRBCS classifiers and the Micro-EBRBS. For the SLAVE and Chi-FRBCS in the case of multi-class datasets, the hypothesis is rejected, which means that the significant differences can be found to show a better accuracy of Micro-EBRBS comparing to the SLAVE and Chi-FRBCS.

Table VII shows the computing time of Micro-EBRBS in comparison with the SLAVE, FH-GBML, FURIA, FARC-HD, and Chi-FRBCS, where the result of the best computing time is highlighted in bold-face and the number in brackets denotes the rank of each classifier. In both of two and multi-class datasets, the computing time of Micro-EBRBS is close to that of ChiFRBCS, and is significantly faster than the other FRBCS classifiers. This is because some additional methodologies were used to improve the FRBCS classifiers, e.g. the genetic algorithm, which is an iterative optimization algorithm, and is one of the components of SLAVE and FARC-HD, leading to a time-consuming process while using those FRBCS classifiers.

Hence, in the application of FRBCS classifiers for addressing big data classification problems, the related works introduced in Section II-B were all based on Chi-FRBCS owning to its high efficient process of dealing with data.

In order to further compare with the Micro-EBRB and ChiFRBCS, Figs. 15 and 16 show their time of rule generation scheme, inference scheme, and total as well as the accuracy over two and multi-class relatively large datasets, respectively.

For the two-class datasets Banknote and Magic, the computing time of rule generation scheme regarding the ChiFRBCS closely approximates to that regarding the Micro- 
TABLE VIII

COMPARISON OF COMPUTING EFFICIENCY FOR MICRO-EBRBS AND CHI-FRBCS

\begin{tabular}{|c|c|c|c|c|c|c|c|c|c|c|}
\hline \multirow{2}{*}{$\begin{array}{l}\text { No. of } \\
\text { classes }\end{array}$} & \multirow[t]{2}{*}{ Dataset } & \multicolumn{2}{|c|}{ Rule generation (ms) } & \multirow[t]{2}{*}{ Ratio } & \multicolumn{2}{|c|}{ Inference $(\mathrm{ms})$} & \multirow[t]{2}{*}{ Ratio } & \multicolumn{2}{|c|}{ Total (ms) } & \multirow[t]{2}{*}{ Ratio } \\
\hline & & Micro-EBRBS & Chi-FRBCS & & Micro-EBRBS & Chi-FRBCS & & Micro-EBRBS & Chi-FRBCS & \\
\hline \multirow[t]{2}{*}{ Two-class } & Banknote & 13.5 & 13.5 & 1.000 & 5.2 & 2.3 & 2.261 & 18.7 & 15.8 & 1.184 \\
\hline & Magic & 303.5 & 237.2 & 1.280 & 843.6 & 767.0 & 1.100 & $1,147.1$ & $1,004.5$ & 1.142 \\
\hline \multirow[t]{2}{*}{ Multi-class } & Waveform & 496.7 & 414.8 & 1.197 & $3,542.1$ & $6,034.2$ & 0.587 & $4,038.8$ & $6,449.0$ & 0.626 \\
\hline & Satimage & $1,386.8$ & $1,162.2$ & 1.193 & $10,864.9$ & $85,759.8$ & 0.127 & $12,251.7$ & $86,922.0$ & 0.141 \\
\hline
\end{tabular}

TABLE IX

COMPARISON OF ACCURACY AND NUMBER OF RULES AND ACTIVATED RULES FOR MICRO-EBRBS AND CHI-FRBC

\begin{tabular}{|c|c|c|c|c|c|c|c|c|c|c|}
\hline \multirow{2}{*}{$\begin{array}{l}\text { No. of } \\
\text { classes }\end{array}$} & \multirow[t]{2}{*}{ Dataset } & \multicolumn{2}{|c|}{ No. of rules } & \multirow[t]{2}{*}{ Ratio } & \multicolumn{2}{|c|}{ No. of activated rules } & \multirow[t]{2}{*}{ Ratio } & \multicolumn{2}{|c|}{ Accuracy (\%) } & \multirow[t]{2}{*}{ Ratio } \\
\hline & & Micro-EBRBS & Chi-FRBCS & & Micro-EBRBS & Chi-FRBCS & & Micro-EBRBS & Chi-FRBCS & \\
\hline \multirow[t]{2}{*}{ Two-class } & Banknote & 30.8 & 30.8 & 1.000 & 22.3 & 12.2 & 1.828 & 97.34 & 94.42 & 1.031 \\
\hline & Magic & 354.1 & 354.1 & 1.000 & 140.9 & 99.3 & 1.419 & 77.38 & 80.62 & 0.960 \\
\hline \multirow[t]{2}{*}{ Multi-class } & Waveform & $2,032.7$ & 2,031.9 & 1.000 & $1,494.2$ & 451.9 & 3.307 & 83.97 & 74.66 & 1.125 \\
\hline & Satimage & $3,182.0$ & $3,182.0$ & 1.000 & 945.6 & 485.6 & 1.947 & 85.41 & 74.79 & 1.142 \\
\hline
\end{tabular}

TABLE X

COMPARISON OF ACCURACY AND NUMBER OF RULES AND ACTIVATED RULES FOR MICRO-EBRBS AND CHI-FRBC

\begin{tabular}{|c|c|c|c|c|c|c|c|c|}
\hline No. of classes & Dataset & $\mathrm{KNN}$ & NB & DT & RF & ANN & SVM & Micro-EBRBS \\
\hline \multirow[t]{6}{*}{ Two-class } & Diabetes & $74.30 \%(6)$ & $76.84 \%$ (4) & $77.61 \%(3)$ & $77.86 \%(2)$ & $79.13 \%(1)$ & $66.92 \%(7)$ & $74.91 \%(5)$ \\
\hline & Cancer & $94.02 \%$ (4) & $93.15 \%(6)$ & $93.32 \%(5)$ & $95.61 \%(3)$ & $96.31 \%(2)$ & $62.74 \%(7)$ & $96.49 \%(1)$ \\
\hline & Transfusion & $76.20 \%$ (4) & $75.40 \%(5)$ & $78.34 \%$ (1) & $72.33 \%(7)$ & $76.34 \%$ (3) & $75.27 \%(6)$ & $76.52 \%(2)$ \\
\hline & Banknote & $92.93 \%(5)$ & $84.26 \%(7)$ & $90.31 \%(6)$ & $99.20 \%(2)$ & $98.25 \%(3)$ & $100.00 \%(1)$ & $97.34 \%(4)$ \\
\hline & Magic & $74.79 \%(5)$ & $72.69 \%(6)$ & $81.17 \%$ (3) & $86.14 \%$ (1) & $83.73 \%(2)$ & $65.88 \%(7)$ & $77.38 \%(4)$ \\
\hline & Average rank & 4.8 & 5.6 & 3.6 & 3.0 & 2.2 & 5.6 & 3.2 \\
\hline \multirow[t]{6}{*}{ Multi-class } & Wine & $97.19 \%(1.5)$ & $96.63 \%(3)$ & $92.13 \%(6)$ & $94.38 \%(5)$ & $97.19 \%(1.5)$ & $44.38 \%(7)$ & $95.84 \%$ (4) \\
\hline & Waveform & $82.88 \%$ (4) & $81.02 \%(5)$ & $73.26 \%(7)$ & $80.20 \%(6)$ & $85.78 \%$ (2) & $86.10 \%(1)$ & $84.00 \%$ (3) \\
\hline & Glass & $61.21 \%(5)$ & $47.66 \%(6)$ & $67.76 \%$ & $71.50 \%$ & $39.72 \%(7)$ & $69.16 \%(2)$ & $63.32 \%(4)$ \\
\hline & Red Wine & $57.22 \%(5)$ & $54.97 \%(7)$ & $58.16 \%(3)$ & $64.35 \%(1)$ & $57.04 \%(6)$ & $57.85 \%(4)$ & $58.36 \%(2)$ \\
\hline & Satimage & $74.34 \%(6)$ & $79.70 \%$ (4) & $79.63 \%(5)$ & $88.94 \%$ (1) & $86.28 \%(2)$ & $26.08 \%(7)$ & $85.41 \%(3)$ \\
\hline & Average rank & 4.3 & 5.0 & 4.8 & 2.8 & 3.7 & 4.2 & 3.2 \\
\hline
\end{tabular}

TABLE XI

FRIEDMAN AND HOLM TESTS TO COMPARE THE ACCURACY OF MICRO-EBRBS AND SIX MACHINE-LEARNING CLASSIFIERS $(\alpha=0.1)$

\begin{tabular}{llllllll}
\hline \hline Item & Indicator & KNN & NB & DT & RF & ANN & SVM \\
\hline Two-class & p value & 0.2416 & 0.0790 & 0.7697 & 0.8836 & 0.4642 & 0.0790 \\
& Critical value & 0.0250 & 0.0167 & 0.0500 & 0.1000 & 0.0333 & 0.0167 \\
& Hypothesis & Accepted & Accepted & Accepted & Accepted & Accepted & Accepted \\
Multi-class & p value & 0.4208 & 0.1877 & 0.2416 & 0.7697 & 0.7144 & 0.4642 \\
& Critical value & 0.0250 & 0.0167 & 0.020 & 0.1000 & 0.0500 & 0.0333 \\
& Hypothesis & Accepted & Accepted & Accepted & Accepted & Accepted & Accepted \\
\hline \hline
\end{tabular}

EBRBS from 2-CV to 10-CV. Meanwhile, for the dataset Banknote, the Micro-EBRBS is slightly better than the ChiFRBCS in terms of the computing time of inference scheme and the accuracy. But for the dataset Magic, the Chi-FRBCS is slightly better than the Micro-EBRBS regarding the computing time and accuracy. For the multi-class datasets Waveform and Satimage, apart from the computing time of rule generation scheme, the Micro-EBRBS is much better than the Chi-FRBCS in term of the computing time of the inference scheme and the accuracy.

In order to show the detailed comparison of Micro-EBRBS and Chi-FRBCS, Tables VIII and IX provide the results and their ratio obtained from $10-\mathrm{CV}$, such as the rule generation time, inference time, total time, number of rules, number of activated rules, and accuracy. Table VIII shows that the ChiFRBCS has less computing time than the Micro-EBRBS in terms of the rule generation scheme for both two and multiclass datasets and the inference scheme for two-class datasets. However, the Micro-EBRBS has less computing time of the inference scheme than the Chi-FRBCS in the multi-class datasets. As a result, the Chi-FRBCS has better total time and their ratio are 1.184 and 1.142 for the two-class datasets Banknote and Magic, and the Micro-EBRBS has better total time and their ratio are 0.626 and 0.141 for the multi-class datasets Waveform and Satimage.

Table IX shows that the number of rules in the Chi-FRBCS is the same as the Micro-EBRBS, but the number of activated rules in the Chi-FRBCS is smaller than the Micro-EBRBS, where the maximum ratio is obtained from the multi-class dataset Waveform and its ratio is 3.307, and except for the dataset Magic, the accuracy of the Chi-FRBCS is worse than the Micro-EBRBS for all two and multi-class datasets, where the maximum ratio is obtained from the multi-class dataset Satimage and its ratio is 1.142 .

In order to compare the accuracy of the Micro-EBRBS with the conventional machine-learning classifiers, Table $\mathrm{X}$ shows the accuracy of seven classifiers for ten classification datasets. The number in brackets denotes the rank of each classifier and the best result is marked as bold in Table X. For the two-class datasets, the accuracy of the RF and ANN are better than the Micro-EBRBS ranked at the 3rd place, and are further better than the KNN, NB, DT, and SVM. For the multi-class dataset, 


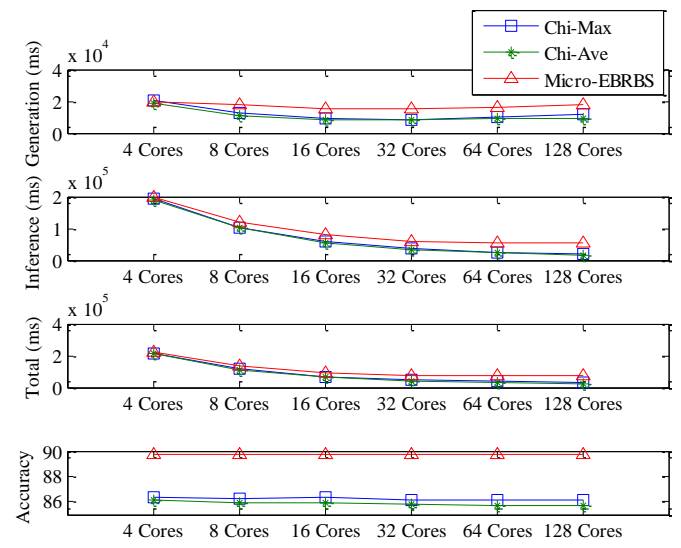

(a) Census (two classes)

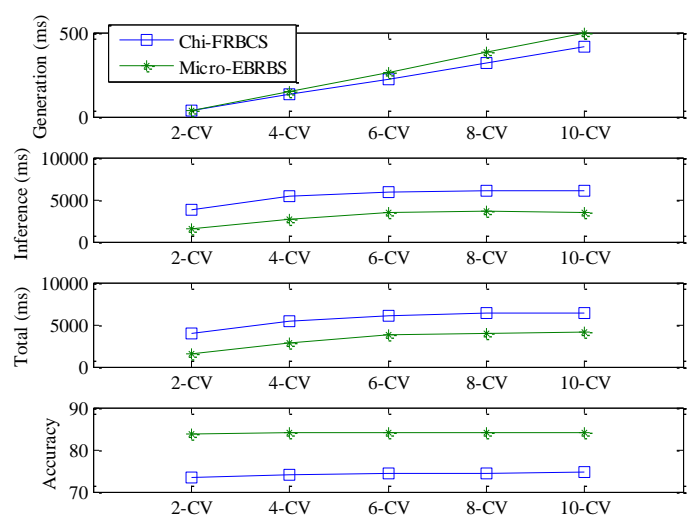

(c) Covtype (seven classes)

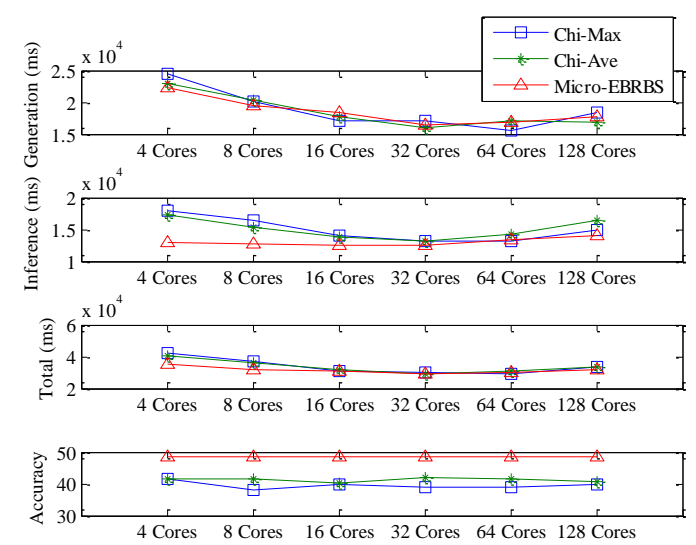

(b) Gas sensors (three classes)
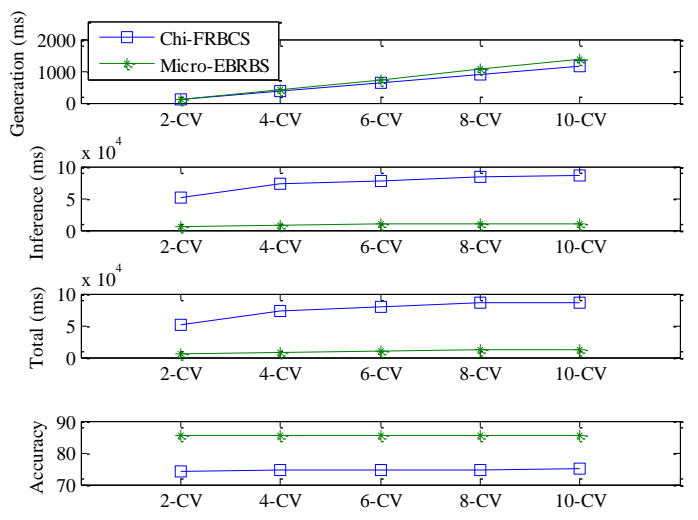

(d) Poker (ten classes)

Fig. 17. Comparison of computing efficiency and accuracy for Chi-Max, Chi-Ave, and Micro-EBRBS

the Micro-EBRBS can reach the second best average rank better than the KNN, DT, NB, ANN, and SVM. From Table X, RF obtains the best average rank in both two and multi-class datasets. This is partly because RF is an ensemble learning method that operates by constructing a multitude of DTs. Hence, in one sense, the ensemble learning used in the RF can also use to improve the Micro-EBRB.

Table XI shows the statistical analysis of accuracy while the Micro-EBRBS is selected as the control method for the Friedman and Holm tests. From Table XI, all hypotheses regarding the six machine-learning classifiers are accepted, namely the Micro-EBRBS and the machine-learning classifiers have a similar accuracy for two and multi-class datasets without significant differences.

In summary, for the comparison of the Micro-EBRBS and the conventional classifiers, the experiment results have proven that the Micro-EBRBS has the similar accuracies with the conventional FRBCS and the conventional machine-learning classifiers but its computing time is much less than the conventional FRBCS classifiers except for the Chi-FRBCS.

It is worth noting that the Micro-EBRBS has the higher accuracy but less computing time than the Chi-FRBCS for multi-class datasets.

\section{Comparative analysis between Micro-EBRBS and big data FRBCS classifiers}

The third experiment aims to compare the accuracy and the computing efficiency of the Micro-EBRBS with the big data FRBCS classifiers, where the core supporting theory of the big data FRBCS classifiers is shown in Section IV-C and they include the following two versions [29]:

(1) Chi-FRBCS-BigData-Max (Chi-Max for short): In this big data classifier, the rule generation scheme searches for the fuzzy rules with the same fuzzy label. Among these fuzzy rules, only the fuzzy rule with the highest rule weight is maintained in the fuzzy rule base.

(2) Chi-FRBCS-BigData-Ave (Chi-Ave for short): In this big data classifier, the rule generation scheme also searches for the fuzzy rules with the same fuzzy label. Afterwards, the average rule weight of the fuzzy rules that have the same class is computed. Finally, the fuzzy rules with the greatest average rule weight is kept in the fuzzy rule base.

Fig. 17 shows the computing time and the accuracy of the Micro-EBRBS in comparison with the big data FRBCS classifiers, including Chi-Max and Chi-Ave, over two-class and multi-class datasets, respectively.

From Fig. 17, the Micro-EBRBS has the significantly better computing efficiency for the seven-class dataset Covtype and the ten-class dataset Poker, similar one for the three-class 
TABLE XII

COMPARISON OF COMPUTING TIME AND ACCURACY FOR CHI-MAX, CHI-AVE AND MICRO-EBRBS UNDER FOUR CORES

\begin{tabular}{|c|c|c|c|c|c|c|c|c|c|}
\hline \multirow[t]{2}{*}{ Indicator } & \multirow[t]{2}{*}{ Classifier } & \multicolumn{2}{|c|}{ Census } & \multicolumn{2}{|c|}{ Gas sensors } & \multicolumn{2}{|c|}{ Covtype } & \multicolumn{2}{|c|}{ Poker } \\
\hline & & Value & Ratio & Value & Ratio & Value & Ratio & Value & Ratio \\
\hline \multirow[t]{3}{*}{ Rule generation (ms) } & Micro-EBRBS & 19,817 & - & 22,269 & - & 50,062 & - & 36,099 & 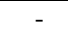 \\
\hline & Chi-Max & 20,007 & 0.991 & 24,349 & 0.915 & 68,246 & 0.734 & 388,942 & 0.093 \\
\hline & Chi-Ave & 18,759 & 1.056 & 23,000 & 0.968 & 67,656 & 0.740 & 376,419 & 0.096 \\
\hline \multirow[t]{3}{*}{ Inference (ms) } & Micro-EBRBS & 199,324 & - & 12,879 & - & 129,858 & - & $1,818,235$ & - \\
\hline & Chi-Max & 191,375 & 1.042 & 17,845 & 0.722 & $8,310,398$ & 0.016 & $141,730,759$ & 0.013 \\
\hline & Chi-Ave & 190,816 & 1.045 & 17,223 & 0.748 & $8,307,228$ & 0.016 & $139,971,396$ & 0.013 \\
\hline \multirow[t]{3}{*}{ Total (ms) } & Micro-EBRBS & 219,141 & - & 35,148 & - & 179,920 & - & $1,854,334$ & - \\
\hline & Chi-Max & 211,382 & 1.037 & 42,194 & 0.833 & $8,378,644$ & 0.022 & $142,119,701$ & 0.013 \\
\hline & Chi-Ave & 209,575 & 1.046 & 40,223 & 0.874 & $8,374,884$ & 0.022 & $140,347,815$ & 0.013 \\
\hline \multirow[t]{3}{*}{ Accuracy (\%) } & Micro-EBRBS & 89.69 & - & 48.60 & - & 70.24 & - & 57.20 & - \\
\hline & Chi-Max & 86.34 & 1.039 & 41.34 & 1.176 & 65.19 & 1.078 & 52.79 & 1.084 \\
\hline & Chi-Ave & 86.14 & 1.041 & 41.68 & 1.166 & 67.08 & 1.047 & 53.53 & 1.069 \\
\hline
\end{tabular}

TABLE XIII

FRIEDMAN AND HOLM TESTS TO COMPARE THE ACCURACY OF Micro-EBRBS, CHI-MAX, AND CHI-Ave ( $\alpha=0.1$ )

\begin{tabular}{ccc}
\hline \hline Indicator & Chi-Max & Chi-Ave \\
\hline p value & 0.0133 & 0.0771 \\
Critical value & 0.05 & 0.1 \\
Hypothesis & Rejected & Rejected \\
\hline \hline
\end{tabular}

dataset Gas sensors and worse one for the two-class dataset Census comparing to big data FRBCS classifiers. For the ten, seven, and three-class datasets, the computing efficiency differences between these classifiers diminish gradually from 4 cores to 128 cores. Additionally, from the datasets Census and Gas sensors, while 128 cores are used in the cluster computing, the computing time is more than the result obtained from 64 cores mainly because of the increasing cluster costs. So, determination of the number of cores is important to improve the computing efficiency of the Micro-EBRBS.

From the comparison of accuracy, the Micro-EBRBS reflects a better robustness than the big data FRBCS classifiers because the accuracy is consistent for all two and multi-class datasets when varying the number of cores used in the cluster computing, but the accuracy of the big data FRBCS classifiers are changeable. Therefore, comparing to the big data FRBCS classifiers, the Micro-EBRBS is able to provide exactly the same classifier while implementing by the Apache Spark. More specifically, Table XII shows the value and ratio of the MicroEBRBS, Chi-Max, and Chi-Ave under 4 cores, where the best result is marked as bold.

In term of the computing time, the big data FRBCS classifiers have a slight advantage while addressing the twoclass dataset Census and all ratios of rule generation, inference, and total time are less than 1.056. However, the computing time of the Micro-EBRBS is much better than both Chi-Max and Chi-Ave while increasing number of classes, i.e. ten-class dataset Poker whose ratios are all smaller than 0.1 for the rule generation, inference, and total time. In term of the accuracy, the Micro-EBRBS is better than the big data FRBCS classifiers and the range of the ratio can be expressed as [1.039, 1.176] for four datasets.

Additionally, in order to detect significant differences among the accuracy of the Micro-EBRBS, Chi-Max, and Chi-Ave, the Friedman and Holm tests are carried out. Table XIII shows that two hypotheses are rejected because there are significant differences among the obtained results with a level of significance of $\alpha=0.1$. Hence, in the datasets Census, Gas sensors, Covtype, and Poker, the accuracy of the MicroEBRBS is better than that of the Chi-Max and Chi-Ave.

In summary, according to the comparison of the MicroEBRBS and the big data FRBCS classifiers, it is evident that the Micro-EBRBS has the advantage of using less computing time and obtaining better accuracy and robustness for the big data multi-class datasets.

\section{E. Time complexity Comparison}

In this subsection, a comparison of the Micro-EBRBS, EBRBS, and Chi-FRBCS is provided to show which one has a better time complexity to deal with big data multi-class classification problems.

Suppose that there are $L$ rules in EBRB (or sample data), $\bar{L}$ rules in the reduced EBRB, $S$ testing data, $M$ antecedent attribute with $J_{i}$ reference values, and $N$ classes. The time complexity of different schemes in the EBRBS, Micro-EBRBS, and Chi-FRBCS is shown in Table XIV based on discussions in Section II-B, Section III-C, and [29]. Additionally, in order to clearly compare three classifiers, their time complexity can be simplified under the assumptions: (1) the number of sample data $L$ is much bigger than the square of the number of classes $N^{2}$; and (2) the total number of reference values for antecedent attributes $\sum_{i=1}^{M} J_{i}$ is much bigger than the number of classes $N$.

Remark 11. It is clear from Table XIV that the time complexity of the Micro-EBRBS is better than the EBRBS in both the rule generation and the inference schemes due to the following three reasons:

(1) The Micro-EBRBS has a simple process in the rule generation scheme because of excluding the calculation of rule weights comparing to the EBRBS.

(2) The Micro-EBRBS has less number of rules in the reduced EBRB owning to using the proposed rule reduction method to downsize the EBRB comparing to the EBRBS.

(3) The Micro-EBRBS can be implemented by using the Apache Spark to generate rules and classify test input data in parallel thanks to the better performance of independence in the rule generation and the inference schemes

Remark 12. It is clear from Table XIV that the Chi-FRBCS is more efficient than the Micro-EBRBS in the rule generation 
TABLE XIV

TIME COMPLEXITY OF EBRBS, MICRO-EBRBS, AND CHI-FRBCS

\begin{tabular}{ccccc}
\hline \hline & & & Rule generation scheme \\
$\left(L>>N^{2}\right)$ & $\begin{array}{c}\text { Inference scheme } \\
\left(\sum_{i=1}^{M} J_{i}>>>N\right)\end{array}$ \\
\hline EBRBS & $O\left(L^{2} \cdot \sum_{i=1}^{M} J_{i}+L^{2} \cdot N\right)$ & $O\left(S \cdot L \cdot\left(\sum_{i=1}^{M} J_{i}+N\right)\right)$ & $O\left(L^{2} \cdot \sum_{i=1}^{M} J_{i}+L^{2} \cdot N\right)$ & $O\left(S \cdot L \cdot \sum_{i=1}^{M} J_{i}\right)$ \\
Micro-EBRBS & $O\left(L \cdot \bar{L} \cdot \sum_{i=1}^{M} J_{i}+L \cdot \bar{L} \cdot N\right)$ & $O\left(S \cdot \bar{L} \cdot\left(\sum_{i=1}^{M} J_{i}+N\right)\right)$ & $O\left(L \cdot \bar{L} \cdot \sum_{i=1}^{M} J_{i}+L \cdot \bar{L} \cdot N\right)$ & $O\left(S \cdot \bar{L} \cdot \sum_{i=1}^{M} J_{i}\right)$ \\
Chi-FRBCS & $O\left(L \cdot \sum_{i=1}^{M} J_{i}+L \cdot \bar{L}+N^{2} \cdot \bar{L}\right)$ & $O\left(S \cdot \bar{L} \cdot \sum_{i=1}^{M} N^{2}\right)$ & $O\left(L \cdot \sum_{i=1}^{M} J_{i}+L \cdot \bar{L}\right)$ & $O\left(S \cdot \bar{L} \cdot \sum_{i=1}^{M} N^{2}\right)$ \\
\hline \hline
\end{tabular}

scheme. However, the Micro-EBRBS is more efficient than the Chi-FRBCS in the inference scheme, especially for the multiclass classification problems. Additionally, the computing efficiency of both the two big data classifiers can be further improved by using the Apache Spark.

\section{CONCLUSIONS}

In this study, the analysis of the rule weight calculation and the ER algorithm were carried out to investigate the approach of reducing the time complexity of the EBRBS, a popular advanced rule-based system, followed by a ER-C algorithm and a domain division-based rule reduction method proposed for developing a micro version of EBRBS with high computing efficiency, called Micro-EBRBS. Furthermore, the Apache Spark was introduced to implement the Micro-EBRBS for better dealing with big data multi-class classification problems. 14 classification datasets were used to validate the accuracy and computing efficiency of the Micro-EBRBS in comparison with the EBRBS, the conventional FRBCS and machine-learning classifiers, and the big data FRBCS classifiers. The detailed contributions are summarized as follows:

(1) The non-necessity of the rule weight calculation and the ER algorithm involved in the EBRBS were investigated, in which the former demonstrates that it is unnecessary to calculate rule weights for each EBR under the assumption of large amount of data, the latter prove that the ER-C algorithm has the same functionality as the ER algorithm under the assumption of classification problems.

(2) The division point and division domain were defined to divide the input space of the EBRBS into multiple local input spaces. Accordingly, the rule clustering strategy and rule reduction strategy were further defined to propose a domain division-based rule reduction method to downsize EBRB.

(3) The Micro-EBRBS, which includes the rule reduction and the ER-C algorithm but excludes the rule weight calculation in comparison with the EBRBS, and its implementation based on the Apache Spark were developed to deal with big data multiclass classification problems, which were then validated through the detailed case studies. The results have shown advantages of the Micro-EBRBS over the existing methods in terms of computing efficiency and classification accuracy.

For the future research, the application of Micro-EBRBS and further improvement to make it more effective to deal with the practical problem with uncertain and imbalance data.

\section{APPENDIX A. INFERENCE SCHEME OF EBRBS FOR CLASSIFICATION PROBLEMS}

The inference scheme of the EBRBS mainly includes two steps [22]: (1) calculation of activation weights for each EBR using distance measure and (2) integration of activated rules for estimating classes using the ER algorithm.

One thing to note is that the procedure of the first step is the same as the Micro-EBRBS. Hence, the activation weight $w_{k}$ $(k=1, \ldots, L)$ can be obtained by using Eq. (23), which shows a positive correlation between the $w_{k}$ and rule weight $\theta_{k}$. After calculating activation weights, all activated rules should be integrated using the analytical ER algorithm [32], [37]:

$$
\begin{aligned}
\beta_{n}= & \left(\prod_{k=1}^{L}\left(w_{k} \beta_{n, k}+1-w_{k} \sum_{i=1}^{N} \beta_{i, k}\right)-\right. \\
& \left.\prod_{k=1}^{L}\left(1-w_{k} \sum_{i=1}^{N} \beta_{i, k}\right)\right) / \\
& \left(\sum_{i=1}^{N} \prod_{k=1}^{L}\left(w_{k} \beta_{i, k}+1-w_{k} \sum_{j=1}^{N} \beta_{j, k}\right)-\right. \\
& \left.(N-1) \prod_{k=1}^{L}\left(1-w_{k} \sum_{j=1}^{N} \beta_{j, k}\right)-\prod_{k=1}^{L}\left(1-w_{k}\right)\right)
\end{aligned}
$$

The integrated belief distribution of the test input data $\boldsymbol{x}$ is:

$$
f(\boldsymbol{x})=\left\{\left(D_{n}, \beta_{n}\right) ; n=1, \ldots, N\right\}
$$

For classification problems, suppose $D_{n}$ denotes the $n$th class, the estimated class of the EBRBS can be obtained by seeking the greatest belief degree.

$$
f(\boldsymbol{x})=D_{n}, n=\arg \max _{i=1, \ldots, N}\left\{\beta_{i}\right\}
$$

APPENDIX B. BELIEF DISTRIBUTION GENERATION AND RULE WEIGHT CALCULATION OF EBRBS

The belief distribution generation and the rule weight calculation are important processes in the rule generation scheme of the EBRBS and their details can be refer to [22].

One thing to note is that the detailed procedure of the belief distribution generation is the same as the Micro-EBRBS. Hence, for the $k$ th $(k=1, \ldots, L)$ EBR, the belief distributions of the $i$ th antecedent attribute $S_{i}^{k}=\left\{\left(A_{i, j}, \alpha_{i, j}^{k}\right) ; j=1, \ldots, J_{i}\right\}$ $(i=1, \ldots, M)$ and the consequent attribute $S^{k}=\left\{\left(D_{n}, \beta_{n, k}\right)\right.$; $n=1, \ldots, N\}$ can be obtained by using Eqs. (16) and (19).

Definition B.1 (Similarity of two belief distributions). Suppose two belief distribution $P=\left(p_{1}, \ldots, p_{T}\right)$ and $Q=\left(q_{1}, \ldots\right.$, $q_{3}$ ), then the similarity of $P$ and $Q$ can be calculated as follows: 


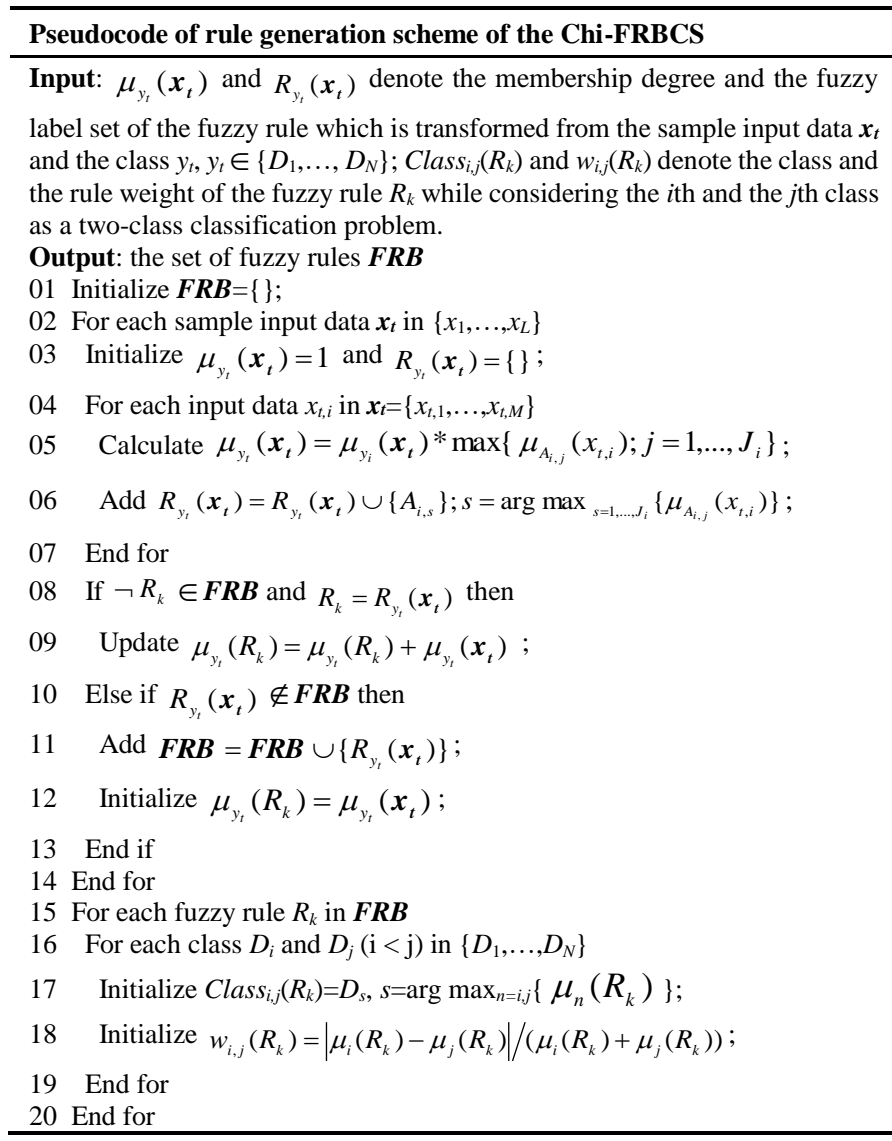

$$
\begin{aligned}
\operatorname{Sim}(P, Q) & =1-d(P, Q) \\
& =1-\min \left\{1, \sqrt{\sum_{t=1}^{T}\left(p_{t}-q_{t}\right)^{2}}\right\}
\end{aligned}
$$

where $d(P, Q)$ denotes the distance between $P$ and $Q$.

Based on Definition B.1, for the $k$ th $(k=1, \ldots, L)$ EBR, the similarity of rule antecedent (SRA) and the similarity of rule consequent (SRC) can be calculated as follows:

$$
\begin{aligned}
\operatorname{SRA}\left(R_{l}, R_{k}\right)= & \min _{t=1, \ldots, M}\left\{\operatorname{Sim}\left(S_{t}^{l}, S_{t}^{k}\right)\right\} \\
= & \min _{t=1, \ldots, M}\left\{1-\min \left\{1, \sqrt{\sum_{j=1}^{J_{t}}\left(\alpha_{t, j}^{l}-\alpha_{t, j}^{k}\right)^{2}}\right\}\right\} \\
\operatorname{SRC}\left(R_{l}, R_{k}\right) & =\operatorname{Sim}\left(S^{l}, S^{k}\right) \\
& =1-\min \left\{1, \sqrt{\sum_{n=1}^{N}\left(\beta_{n, l}-\beta_{n, k}\right)^{2}}\right\} ;
\end{aligned}
$$

where $l=1, \ldots, L$ and $l \neq k ; S_{i}{ }^{k}$ denotes the belief distribution of the $t$ th antecedent attribute in the $k$ th EBR; $S^{k}$ denotes the belief distribution of the consequent attribute in the $k$ th EBR.

Definition B.2 (Consistency of EBRs). Suppose the SRA and the SRC of the $l$ th $(l=1, \ldots, L)$ and the $k$ th $(k=1, \ldots, L ; k \neq l)$ EBRs are $S R A\left(R_{l}, R_{k}\right)$ and $\operatorname{SRC}\left(R_{l}, R_{k}\right)$, respectively, then the consistency of the rules $R_{l}$ and $R_{k}$ can be calculated as follows:

\section{Pseudocode of inference scheme of the Chi-FRBCS}

Input: $S_{i, j}\left(\boldsymbol{x}_{\boldsymbol{t}}\right)$ denotes the score of test input data $\boldsymbol{x}_{\boldsymbol{t}}$ while considering the $i$ th and the $j$ th class as a two-class classification problem; $\mu\left(\boldsymbol{x}_{\boldsymbol{t}}\right)$ denotes the membership degree of the test input data $\boldsymbol{x}_{\boldsymbol{t}} ; w_{i, j}\left(R_{k}\right)$ denotes the rule weight of the fuzzy rule $R_{k}$ while considering the $i$ th and the $j$ th class as a two-class classification problem.

Output: The estimated class $\operatorname{Class}\left(\boldsymbol{x}_{\boldsymbol{t}}\right)(t=1, \ldots, S)$

01 For each test input data $\boldsymbol{x}_{\boldsymbol{t}}$ in $\left\{\boldsymbol{x}_{1}, \ldots, \boldsymbol{x}_{S}\right\}$

02 Initialize $S_{i, j}\left(\boldsymbol{x}_{\boldsymbol{t}}\right)=0(i, j=1, \ldots, N)$

03 For each $D_{i}$ and $D_{j}(i<j)$ in $\left\{D_{1}, \ldots, D_{N}\right\}$

$04 \quad$ For each fuzzy rule $R_{k}$ in $\boldsymbol{F R B}$

$05 \quad$ Initialize $\mu\left(\boldsymbol{x}_{\boldsymbol{t}}\right)=1$;

$06 \quad$ For each input data $x_{t, m}$ in $\boldsymbol{x}_{t}=\left\{x_{t, 1}, \ldots, x_{t, M}\right\}$

$07 \quad$ Calculate $\mu\left(\boldsymbol{x}_{\boldsymbol{t}}\right)=\mu\left(\boldsymbol{x}_{\boldsymbol{t}}\right) * \mu_{A_{m}^{k}}\left(x_{t, m}\right)$;

$08 \quad$ End for

09 If Class $_{i, j}\left(R_{k}\right)=D_{i}$ and $S_{i, j}\left(\boldsymbol{x}_{t}\right)<\mu\left(\boldsymbol{x}_{t}\right) * w_{i, j}\left(R_{k}\right)$ then

$10 \quad$ Calculate $S_{i, j}\left(\boldsymbol{x}_{\boldsymbol{t}}\right)=\mu_{y_{t}}\left(\boldsymbol{x}_{\boldsymbol{t}}\right) * w_{i, j}\left(R_{k}\right)$;

11 Else if $\operatorname{Class}_{i, j}\left(R_{k}\right)=D_{j}$ and $S_{j, i}\left(\boldsymbol{x}_{\boldsymbol{t}}\right)<\mu\left(\boldsymbol{x}_{\boldsymbol{t}}\right) * w_{i, j}\left(R_{k}\right)$ then

$12 \quad$ Calculate $S_{j, i}\left(\boldsymbol{x}_{\boldsymbol{t}}\right)=\mu\left(\boldsymbol{x}_{\boldsymbol{t}}\right) * w_{i, j}\left(R_{k}\right)$;

13 End if

14 End for

15 End for

16 Calculate $\operatorname{Class}\left(\boldsymbol{x}_{\boldsymbol{t}}\right)=D_{n} ; n=\arg \max _{i=1, \ldots, N}\left\{\sum_{j=1}^{N} S_{i, j}\left(\boldsymbol{x}_{\boldsymbol{t}}\right)\right\}$

17 End for

$$
\operatorname{Cons}\left(R_{l}, R_{k}\right)=\exp \left\{-\frac{\left(\frac{\operatorname{SRA}\left(R_{l}, R_{k}\right)}{\operatorname{SRC}\left(R_{l}, R_{k}\right)}-1\right)^{2}}{\left(\frac{1}{\operatorname{SRA}\left(R_{l}, R_{k}\right)}\right)^{2}}\right\}
$$

Based on Definition B.2, the inconsistency degree of the $k$ th EBR can be calculated as follows:

$$
\begin{aligned}
& \operatorname{Incons}\left(R_{k}\right)=\sum_{l=1, l \neq k}^{L}\left(1-\operatorname{Cons}\left(R_{l}, R_{k}\right)\right) \\
& =\sum_{l=1, l \neq k}^{L}\left(1-\exp \left\{-\frac{\left(\frac{\operatorname{SRA}\left(R_{l}, R_{k}\right)}{\operatorname{SRC}\left(R_{l}, R_{k}\right)}-1\right)^{2}}{\left(\frac{1}{\operatorname{SRA}\left(R_{l}, R_{k}\right)}\right)^{2}}\right\}\right)
\end{aligned}
$$

Finally, the rule weight is calculated as follows:

$$
\theta_{k}=1-\frac{\operatorname{Incons}\left(R_{k}\right)}{\sum_{j=1}^{L} \operatorname{Incons}\left(R_{j}\right)}
$$

\section{APPENDIX C. RULE GENERATION AND INFERENCE SCHEMES OF CHI-FRBCS}

\section{A. Time complexity of the rule generation scheme}

The rule generation scheme of the Chi-FRBCS, which consists of the PCF and OVO, is introduced as follows:

As shown in the pseudocode of the rule generation scheme, the time complexity of calculating $\mu_{y_{t}}\left(\boldsymbol{x}_{t}\right)$ shown in the 5th line and updating the set $\boldsymbol{F R \boldsymbol { B }}$ shown in the 8th to the 13th lines 
is $O\left(\sum_{i-1}^{M} J_{i}\right)$ and $O(\bar{L})$, respectively, for each sample data, where $\bar{L}$ is the number of fuzzy rules. In addition, from the 15 th to the 20th lines, its time complexity is $O\left(\bar{L} \times N^{2}\right)$. As a result, the time complexity of the rule generation scheme involved in the Chi-FRBCS is shown as follows:

$$
O\left(L \times\left(\sum_{i=1}^{M} J_{i}+\bar{L}\right)+\bar{L} \times N^{2}\right)
$$

\section{B. Time complexity of the inference scheme}

The inference scheme of the Chi-FRBCS, which consists of the winning rule strategy and OVO, is introduced as follows:

From the pseudocode of the rule generation scheme, the time complexity of calculating $\mu\left(x_{t}\right)$ shown in the 7th line is $O(M)$ for each test input data, fuzzy rule, and two-class classification problem. Hence, the time complexity of the inference scheme involved in the Chi-FRBCS is shown as follows:

$$
O\left(S \times \bar{L} \times M \times N^{2}\right)
$$

\section{REFERENCES}

[1] Akashdeep, I. Manzoor, and N. Kumar, "A feature reduced intrusion detection system using ANN classifier," Expert Syst. Appl., vol. 88, pp. 249-459, Dec., 2017.

[2] N. Al-Najdawi, M. Biltawi, and S. Tedmori, "Mammogram image visual enhancement, mass segmentation and classification," Appl. Soft Comput., vol. 35, pp. 175-185, Oct., 2015.

[3] J. Alcala-Fdez, R. Alcala, and F. Herrera, "A Fuzzy Association Rule-Based Classification Model for High-Dimensional Problems With Genetic Rule Selection and Lateral Tuning," IEEE Trans. Fuzzy Syst., vol. 19, no. 5, pp. 857-872, Oct. 2011.

[4] A. Kattt, "Spark: Lightning-fast Cluster Computing," Apache spark, Nov. 21, 2011. [Online]. Available: https://amplab.cs.berkeley.edu/projects/ spark-lightning-fast-cluster-computing/

[5] W. Ashlock and S. Datta, "Evolved Features for DNA Sequence Classification and Their Fitness Landscapes," IEEE Trans. Evolut. Comput., vol. 17, no. 2, pp. 185-197, Apr., 2013.

[6] A. Calzada, J. Liu, H. Wang, and A. Kashyap, "A New Dynamic Rule Activation Method for Extended Belief Rule-Based Systems," IEEE Trans. Knowl. Data Eng., vol. 27 no. 4, pp. 880-894, Apr., 2015.

[7] L. L. Chang, Z. J. Zhou, Y. You, L. H. Yang, and Z. G. Zhou, "Belief rule based expert system for classification problems with new rule activation and weight calculation procedures," Inf. Sci., vol. 336, pp. 75-91, 2016.

[8] Z. Chi, H. Yan, and T. Pham, "Fuzzy Algorithms with Applications to Image Processing and Pattern Recognition,” World Scientific, 1996, pp. 139-187.

[9] J. Dean and S. Ghemawat, "MapReduce: simplified data processing on large clusters," Commun. ACM, vol. 51, no. 1, pp. 107-113, 2008.

[10] M. Elkano, M. Galar, J. Sanz, and H. Bustince, "CHI-BD: A Fuzzy Rule-Based Classification System for Big Data classification problems," Fuzzy Sets Syst., vol. 348, no. 1, pp. 75-101, Oct. 2018.

[11] M. Espinilla, J. Medina, A. Calzada, J. Liu, L. Martinez, and C. Nugent, "Optimizing the configuration of a heterogeneous architecture of sensors for activity recognition, using the extended belief rule-based inference methodology," Microprocess Microsy., vol. 52, pp. 381-390, Jul., 2017.

[12] A. Fernández, S. D. Rio, A. Bawakid, and F. Herrera, "Fuzzy rule based classification systems for big data with MapReduce: granularty analysis," Adv. Data Anal. Classi., vol. 11, no. 4, pp. 1-20, Dec., 2017.

[13] A. Fernández, A. Altalhi, S. Alshomrani, and F. Herrera, "Why linguistic fuzzy rule based classification systems perform well in big data applications?" Int. J. Comput. Intell. Syst., vol. 10, pp. 1211-1225, 2017.

[14] S. García, A. Fernández, J. Luengo, and F. Herrera, "Advanced nonparametric tests for multiple comparisons in the design of experiments in computational intelligence and data miningexperimental analysis of power,"Inf. Sci., vol. 180, pp. 2044-2064, 2010.
[15] A. González and R. Pérez, "SLAVE: A Genetic Learning System Based on an Iterative Approach," IEEE Trans. Fuzzy Syst., vol. 7, no. 2, pp. 176-191, Apr., 1999.

[16] J. Hühn and E. Hüllermeier, "FURIA: an algorithm for unordered fuzzy rule induction," Data Min. Knowl. Disc., vol. 19, no. 3, pp. 293-319, Dec., 2009.

[17] H. Ishibuchi, T. Nakashima, and T. Muratam, "A fuzzy classifier system that generates fuzzy if-then rules for pattern classification problems," IEEE Int. Conf. Evolu. Comput., vol. 2, pp. 759-764, Nov./ Dec., 1995.

[18] H. Ishibuchi, T. Yamamoto, and T. Nakashima, "Hybridization of Fuzzy GBML Approaches for Pattern Classification Problems," IEEE Trans. Syst., Man, Cybern., B, Cybern., vol. 35, no. 2, pp. 359-365, Apr., 2005.

[19] L. M. Jiao, T. Denoeux, and Q. Pan, "A Hybrid Belief Rule-Based Classification System Based on Uncertain Training Data and Expert Knowledge," IEEE Trans. Syst., Man, Cybern., Syst., vol. 46, no. 12, pp. 1711-1723, Dec., 2016.

[20] L. M. Jiao, Q. Pan, T. Denœux, Y. Liang, and X. X. Feng, "Belief rule-based classification system: Extension of FRBCS in belief functions framework," Inf. Sci., vol. 309, pp. 26-49, Jul., 2015.

[21] B. Krawczyk, M. Galar, M. Wozniak, H. Bustince, and F. Herrera, "Dynamic ensemble selection for multi-class classification with one-class classifiers," Pattern Recog., vol. 83, pp. 34-51, Nov., 2018.

[22] J. Liu, L. Martinez, A. Calzada, and H. Wang, "A novel belief rule base representation, generation and its inference methodology," Knowl. Based Syst., vol. 53, pp. 129-141, Nov., 2013.

[23] Z. G. Liu, Q. Pan, J. Dezert, J. W. Han, and Y. He, "Classifier Fusion with Contextual Reliability Evaluation," IEEE Trans. Cybern., vol. 48, no. 5, pp. 1605-1618, May, 2018.

[24] Z. G. Liu, Q. Pan, J. Dezert, and A. Martin, "Combination of Classifiers with Optimal Weight Based on Evidential Reasoning," IEEE Trans. Fuzzy Syst., vol. 26, no. 3, pp. 1217-1230, Jun., 2018.

[25] Z. G. Liu, Q. Pan, J. Dezert, and G. Mercier, "Hybrid Classification System for Uncertain Data," IEEE Trans. Syst., Man, Cybern., Syst., vol. 47, no. 10, pp. 2783-2790, Otc., 2017.

[26] V. Lopez, S. D. Rio, J. M. Benitez, and F. Herrera, "Cost-sensitive linguistic fuzzy rule based classification systems under the MapReduce framework for imbalanced big data," Fuzzy Sets Syst., vol. 258, pp. 5-38, Jan., 2015.

[27] A. Lorena, A. Carvalho, and J. Gama, "A review on the combination of binary classifiers in multiclass problems," Artif. Intell. Rev., vol. 30, no. 1-4, pp. 19-37, Dec., 2008.

[28] M. I. Rey, M. Galende, M. J. Fuente, and G. I. Sainz-Palmero, "Multi-objective based Fuzzy Rule Based Systems (FBRSs) for trade-off improvement in accuracy and interpretability: a rule relevance point of view," Knowl. Based Syst., vol. 127, pp. 67-84, Jul., 2017.

[29] S. D. Rio, V. Lopez, J. M. Benitez, and F. Herrera, "A MapReduce Approach to Address Big Data Classification Problems Based on the Fusion of Linguistic Fuzzy Rules," Int. J. Comput. Intell. Syst., vol. 8, no. 3, pp. 422-437, Feb., 2015.

[30] UCI repository of machine learning database, Sep. 14, 2018. [Online]. Available: http://archive.ics.uci.edu/ml/index.php.

[31] L. X. Wang and J.M. Mendel, "Generating fuzzy rules by learning from examples," IEEE Trans. Syst., Man, and Cybern., vol. 22, no. 6, pp. 1414-1427, Nov./Dec., 1992.

[32] Y. M. Wang, J. B. Yang, and D. L. Xu, "Environmental impact assessment using the evidential reasoning approach," Eur. J. Oper. Res., vol. 174, no. 3, pp. 1885-1913, Nov., 2006.

[33] X. J. Xu, C. X. Sheng, C. Q. Yuan, D. L. Xu, and J. B. Yang, "A belief rule-based expert system for fault diagnosis of marine diesel engines," IEEE Trans. Syst., Man, Cybern., Syst., to be published. DOI: 10.1109/ TSMC.2017.2759026.

[34] C. X. Yan, M. N. Luo, H. Liu, Z. H. Li, and Q. H. Zheng, "Top-k multi-class SVM using multiple features," Inf. Sci., vol. 432, pp. 479-494, Mar., 2018.

[35] J. B. Yang, "Rule and utility based evidential reasoning approach for multiattribute decision analysis under uncertainties," Eur. J. Oper. Res., vol. 131, no. 1, pp. 31-61, May, 2001.

[36] J. B. Yang, J. Liu, D. L. Xu, J. Wang, and H. W. Wang, "Optimization models for training belief-rule-based systems," IEEE Trans. Syst., Man, Cybern., A, Syst. Humans, vol. 37, no. 4, pp. 569-585, Jul., 2007.

[37] J. B. Yang, J. Liu, J. Wang, H. S. Sii, and H. W. Wang, "Belief rule-base 
inference methodology using the evidential reasoning approachRIMER," IEEE Trans. Syst., Man, Cybern., A, Syst. Humans, vol. 36, no. 2, pp. 266-285, Mar., 2006.

[38] L. H. Yang, Y. M. Wang, Y. X. Lan, L. Chen, and Y. G. Fu, "A data envelopment analysis (DEA)-based method for rule reduction in extended belief-rule-based systems," Knowl. Based Syst., vol. 123, pp. 174-187, May, 2017.

[39] L. H. Yang, Y. M. Wang, Q. Su, Y. G. Fu, and K. S. Chin, "Multi-attribute search framework for optimizing extended belief rulebased systems," Inf. Sci., vol. 370-371, pp. 159-183, Nov., 2016.

[40] Z. J. Zhou, G. Y. Hu, B. C. Zhang, C. H. Hu, Z. G. Zhou, and P. L. Qiao, "A model for hidden behavior prediction of complex systems based on belief rule base and power set," IEEE Trans. Syst., Man, Cybern., Syst., vol. 99, pp. 1-7, Sep., 2017.

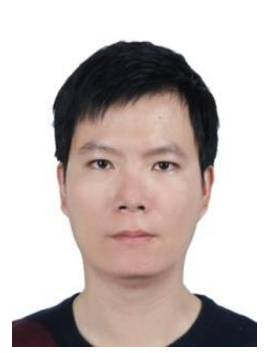

Long-Hao Yang received the B.Eng. and M.Eng. degrees from Fuzhou University, China, in 2012 and 2015, respectively, and he is currently pursuing the $\mathrm{Ph}$.D. degree at Fuzhou University, China.

$\mathrm{He}$ has published over 10 articles in BRB-related studies. His current research interests include BRB learning, machine learning and artificial intelligence, and BRB applications in complex systems modeling.

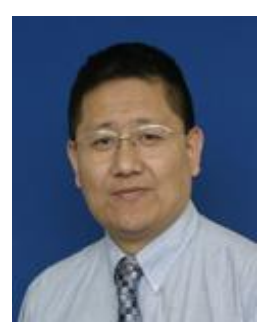

Jun Liu (M'09) received the BSc and MSc degrees in applied mathematics, and the $\mathrm{PhD}$ degree in information engineering from Southwest Jiaotong University, Chengdu, China, in 1993, 1996, and 1999, respectively.

$\mathrm{He}$ is currently a Reader in Computer Science at Ulster University, Northern Ireland, UK. His current research interests include logic and reasoning methods for intelligent systems and formal verification; intelligent DSSs and information management, with applications in health care, engineering, and industry field, etc.; applied computational intelligence for uncertainty analysis and optimisation. He has published more than 80 papers in journals indexed by the SCI and more than 80 contributions in International Conferences.

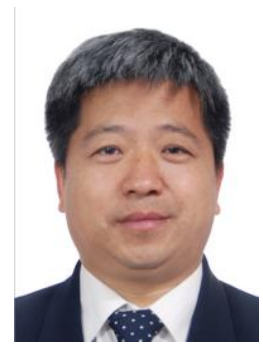

Ying-Ming Wang received his MSc in Systems Engineering from Huazhong University of Science and Technology, China, in 1987, and Ph.D. in Automatic Control Theory and Application from Southeast University, China, in 1991.

$\mathrm{He}$ is currently a full distinguished professor of Changjiang Scholars Program at Fuzhou University, Fuzhou, China. He has published over $123 \mathrm{SCI}$ and 28 SSCI-indexed journal papers and has been the most cited Chinese researchers since 2014. His research interests include multiple criteria decision analysis, data envelopment analysis, rule-based inference, and quality function deployment.

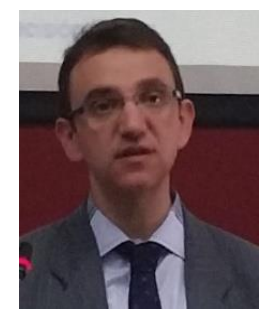

Luis Martínez (M’10) received the M.Sc. and $\mathrm{Ph} . \mathrm{D}$. degrees in computer sciences from the University of Granada, Granada, Spain, in 1993 and 1999, respectively.
He is currently a Full Professor with the Computer Science Department, University of Jaén, Jaén, Spain. He is also Visiting Professor in University of Technology Sydney, University of Portsmouth (Isambard Kingdom Brunel Fellowship Scheme), and in the Wuhan University of Technology (Chutian Scholar), Guest Professor in the Southwest Jiaotong University and Honourable professor in Xihua University both in Chengdu (China). He has co-edited eleven journal special issues on fuzzy preference modelling, soft computing, linguistic decision making and fuzzy sets theory and has been main researcher in $14 \mathrm{R} \& \mathrm{D}$ projects, also has published more than 100 papers in journals indexed by the SCI and more than 150 contributions in International Conferences related to his areas. His current research interests include decision making, fuzzy logic-based systems, computing with words and recommender systems. He was a recipient of the IEEE Transactions on fuzzy systems Outstanding Paper Award 2008 and 2012 (bestowed in 2011 and 2015, respectively). $\mathrm{He}$ is a Co-Editor-in-Chief of the International Journal of Computational Intelligence Systems and an Associate Editor of the journals, including the IEEE Transactions on fuzzy systems, Information Fusion, the International Journal of Fuzzy Systems, the Journal of Intelligent and Fuzzy Systems. He is a member of IEEE and of the European Society for Fuzzy Logic and Technology. Eventually, he has been appointed as Highly Cited Researcher 2017 in Computer Science 